\title{
Fractal approaches to characterize the structure of capillary suspensions using rheology and confocal microscopy
}

Frank Bossler, Johannes Maurath, Katrin Dyhr, Norbert Willenbacher, and Erin Koos

Citation: Journal of Rheology 62, 183 (2018); doi: 10.1122/1.4997889

View online: https://doi.org/10.1122/1.4997889

View Table of Contents: http://sor.scitation.org/toc/jor/62/1

Published by the The Society of Rheology

\section{Articles you may be interested in}

Single polymer dynamics for molecular rheology

Journal of Rheology 62, 371 (2018); 10.1122/1.5013246

Extending yield-stress fluid paradigms

Journal of Rheology 62, 357 (2018); 10.1122/1.5003841

Stress bifurcation in large amplitude oscillatory shear of yield stress fluids Journal of Rheology 62, 89 (2018); 10.1122/1.4986062

Asymmetric soft-hard colloidal mixtures: Osmotic effects, glassy states and rheology Journal of Rheology 62, 63 (2018); 10.1122/1.5009192

Frequency-sweep medium-amplitude oscillatory shear (MAOS)

Journal of Rheology 62, 277 (2018); 10.1122/1.4999795

A constitutive model for simple shear of dense frictional suspensions

Journal of Rheology 62, 457 (2018); 10.1122/1.4999237

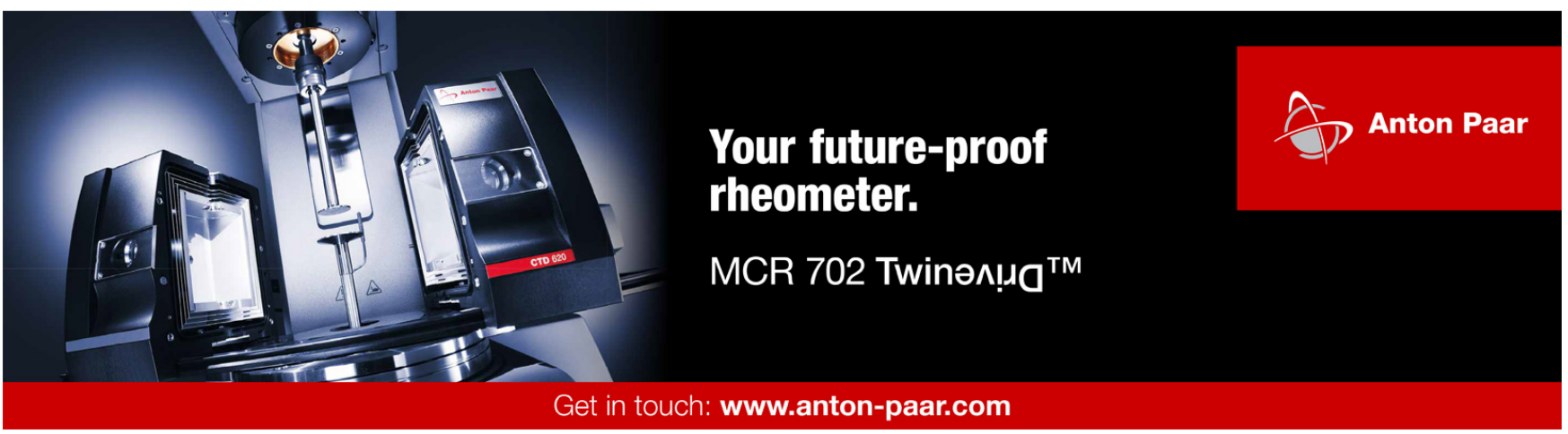




\title{
Fractal approaches to characterize the structure of capillary suspensions using rheology and confocal microscopy
}

\author{
Frank Bossler ${ }^{\text {a) }}$ \\ Karlsruhe Institute of Technology, Institute for Mechanical Process Engineering and Mechanics, \\ Gotthard-Franz-Straße 3, 76131 Karlsruhe, Germany and Department of Chemical Engineering, KU Leuven, \\ Celestijnenlaan 200f, 3001 Leuven, Belgium
}

Johannes Maurath, Katrin Dyhr, and Norbert Willenbacher

Karlsruhe Institute of Technology, Institute for Mechanical Process Engineering and Mechanics, Gotthard-Franz-Straße 3, 76131 Karlsruhe, Germany

Erin Koos

\begin{abstract}
Karlsruhe Institute of Technology, Institute for Mechanical Process Engineering and Mechanics, Gotthard-Franz-Straße 3, 76131 Karlsruhe, Germany and Department of Chemical Engineering, KU Leuven, Celestijnenlaan 200f, 3001 Leuven, Belgium
\end{abstract}

(Received 27 July 2017; final revision received 28 September 2017; published 21 November 2017)

\begin{abstract}
The rheological properties of a particle suspension can be substantially altered by adding a small amount of a secondary fluid that is immiscible with the bulk phase. The drastic change in the strength of these capillary suspensions arises due to the capillary forces, induced by the added liquid, leading to a percolating particle network. Using rheological scaling models, fractal dimensions are deduced from the yield stress and from oscillatory strain amplitude sweep data as function of the solid volume fraction. Exponents obtained using aluminumoxide-based capillary suspensions, with a preferentially wetting secondary fluid, indicate an increase in the particle gel's fractal dimension with increasing particle size. This may be explained by a corresponding relative reduction in the capillary force compared to other forces. Confocal images using a glass model system show the microstructure to consist of compact particle flocs interconnected by a sparse backbone. Thus, using the rheological models, two different fractal dimensionalities are distinguished: a lower network backbone dimension $(D=1.86-2.05)$ and an intrafloc dimension $(D=2.57-2.74)$. The latter is higher due to the higher local solid volume fraction inside of the flocs compared to the sparse backbone. Both of these dimensions are compared with values obtained by analysis of spatial particle positions from three-dimensional confocal microscopy images, where dimensions between 2.43 and 2.63 are computed, lying between the two dimension ranges obtained from rheology. The fractal dimensions determined via this method corroborate the increase in structural compactness with increasing particle size. (C) 2017 The Society of Rheology. https://doi.org/10.1122/1.4997889
\end{abstract}

\section{INTRODUCTION}

Capillary suspensions are ternary solid-liquid-liquid systems. They consist of particle suspensions with a small amount of added secondary fluid that is immiscible with the bulk liquid [1]. The added fluid causes a sample-spanning particle network to form, which leads to a dramatic alteration of the suspension rheology and stability [1-6]. This effect can be used as a simple method to tune the flow behavior of suspensions that would otherwise require the addition of rheology control agents, surfactants, or other additives [7]. Capillary suspensions can be used for various applications such as precursors for ceramic or glass filters with high porosity and simultaneously small pore size [8-10], porous polymeric or hybrid membranes [11,12] improved polymer blends [13-15], slurries for battery electrodes or printable

\footnotetext{
a) Author to whom correspondence should be addressed; electronic mail: frank.bossler@kit.edu

(C) 2017 by The Society of Rheology, Inc.

J. Rheol. 62(1), 183-196 January/February (2018)
}

electronics with accurately molded edge shape, good charge transfer properties and reduced resistivity [16-18], crackfree films [19], thermal interface materials [20], carbon slurries from renewable sources used for energy generation [21], the preparation of novel food products [22,23], or as 3D printing paste formulations [24,25].

Capillary suspensions can be formed either with the secondary fluid wetting better than the bulk phase, which is referred to as pendular state, or wetting less well in the capillary state. In both cases, a percolating particle network arises leading to a transition in the rheological properties due to network formation $[1,26]$. Correspondingly, capillary suspensions can be regarded as attractive particle gels.

The structure of capillary suspensions and their strength depends on the solid particle radius $a$, the interfacial tension $\Gamma$ between the fluids, and the wetting properties of both liquids comprised by the three phase contact angle $\theta$ that the secondary fluid forms toward the particles in a bulk liquid environment [7]. Additionally, the structure and strength are 
determined by the volume and number of the second fluid entities interconnecting the particles [26]. The overall second fluid volume fraction $\phi_{\mathrm{sec}}$ in capillary suspensions is small compared to the volume fraction of the dispersed solid particles $\phi_{\text {solid }}$ and especially to the fraction of bulk liquid [6]. In the pendular state, the capillary force $F_{\mathrm{c}}$ in a concave bridge of volume $V_{\text {bridge }}$ between two equally sized spherical particles can be computed either by solving the YoungLaplace equation or by assuming a certain bridge shape. Typically, the attraction energy between such particles connected by the capillary force is many times higher than thermal energy $k_{\mathrm{B}} T$ [27]. Accordingly, the capillary force is also orders of magnitude higher than the van der Waals force [2] as well as gravity [28]. This leads to an increased cohesion between particles in capillary suspensions compared to suspensions without added secondary fluid that results in the observed marked changes in flow behavior and prevents particle sedimentation [22]. The capillary force of single bridges can be related to the macroscopic stress of a percolated capillary suspension network. The relationship between capillary force and yield stress $\sigma_{\mathrm{y}}$ for equally sized particles in direct contact is given by

$$
\begin{aligned}
\sigma_{\mathrm{y}} & =f\left(\phi_{\text {solid }}, \phi_{\text {sec }}\right) \frac{F_{c}}{a^{2}} \\
& =f\left(\phi_{\text {solid }}, \phi_{\text {sec }}\right) g\left(\frac{V_{\text {bridge }}}{a^{3}}\right) \frac{2 \pi \Gamma \cos \theta}{a}
\end{aligned}
$$

with $f\left(\phi_{\text {solid }}, \phi_{\text {sec }}\right)$ being a function of particle and second fluid volume fractions and $g\left(V_{\text {bridge }} / a^{3}\right)$ a function of the bridge volume relative to the particle volume [2,29-31]. While $V_{\text {bridge }}$ is obviously related to the second fluid volume fraction $\phi_{\mathrm{sec}}$, it also strongly depends on the sample preparation method [13,32] and contact angle [26]. Such a dependence on sample preparation conditions is true of capillary suspensions and other strong attractive particle gels that are not in thermodynamic equilibrium since the structure does not organize itself in a reproducible manner without the application of well-defined external forces [33].

The structure of attractive particle gels is often treated as a random fractal and thus is described by the fractal dimension $D$. The aim of this study is to apply fractal approaches to capillary suspensions and the paper is organized as follows: In Sec. II, we discuss the concept of fractal dimensions and summarize different approaches to calculate the fractal dimension of particle networks from rheological scaling laws or image analysis. After having described our materials and methods in Sec. III, our first study (Sec. IV A) evaluates the yield stress of aluminum oxide based capillary suspensions as a function of particle size as well as secondary and solid volume fractions. We then apply rheological scaling laws to evaluate fractal dimensions in Sec. IV B. In Sec. IV C, we deduce fractal dimensions from image analysis of the structure using a series of confocal microscopy experiments with capillary suspensions based on fluorescently labeled silica beads. Finally, we compare the approaches and summarize our findings in the conclusions of Sec. V.

\section{THEORETICAL BACKGROUND}

The fractal dimension $D$ is a parameter to describe the internal geometry of particle clusters. When fractal clusters consisting of $N$ particles are formed by aggregation, the cluster radius $R_{\mathrm{c}}$ scales as

$$
N \propto R_{\mathrm{c}}^{D}
$$

where $D$ in three-dimensional space can take values of $1 \leq D \leq 3[34,35]$. Counting the number of particles inside a sphere of radius $R$ and transforming Eq. (2) to nondimensional form by relating the radial coordinate $R$ to single particle radius $a$ directly leads to

$$
N\left(\frac{R}{a}\right) \propto\left(\frac{R}{a}\right)^{D}
$$

where $N(R / a)$ is the particle number as function of the normalized radial coordinate $R / a$ and the center of gyration of the cluster is defined as $R=0$ [36]. In a real system, there is some variability in the size of the particles, the size of the aggregate, and the fractal dimension for each cluster, and so these values should be taken as the mean values for the observed sample.

Different models have classically been used to describe cluster formation kinetics. The diffusion limited (DLCA) and reaction limited cluster aggregation (RLCA) models are distinguished by the sticking probability of each additional particle contacting the cluster. The sticking probability is 1 (every particle sticks to the cluster upon first contact) for DLCA, while it is close to 0 for RLCA (a particle can contact the cluster many times before it finally adheres) [37]. Thus, RLCA clusters have a more densely packed structure than DLCA which is captured by their fractal dimension values. Typically, DLCA clusters have $D \approx 1.85 \pm 0.1$, while $D \approx 2.1 \pm 0.1$ for RLCA in three-dimensional space $[37,38]$. However, this dimension can depend on a variety of other parameters, such as external shear $[39,40]$.

Experimental determination of $D$ by Eq. (3) is quite straightforward using 3D confocal images of the clustered particles from which the particle positions can be calculated $[26,41]$. There are some caveats, however, when a percolated particle network instead of a single particle cluster is evaluated. In this case, different fractal clusters touch and merge into each other. Thus, adjacent clusters that were initially grown separately share particles with each other and the corresponding structure in the overlap regions can no longer be well described by the cluster fractal dimension. The correlation length $\xi$, which denotes the maximum length scale before a cluster loses its internal fractality, is a helpful tool to capture this critical overlap. Therefore, Eqs. (2) and (3) are limited to $R \leq \xi$. Figure 1 shows an idealized drawing of a particle gel originating from merged clusters where $\xi$ is labeled. The correlation length decreases as the number density of particles is increased. For attractive particle gels, $\xi$ can be directly related to the volume fraction of particles

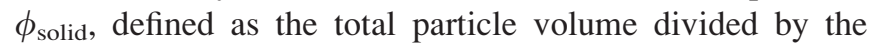
total sample volume, with 


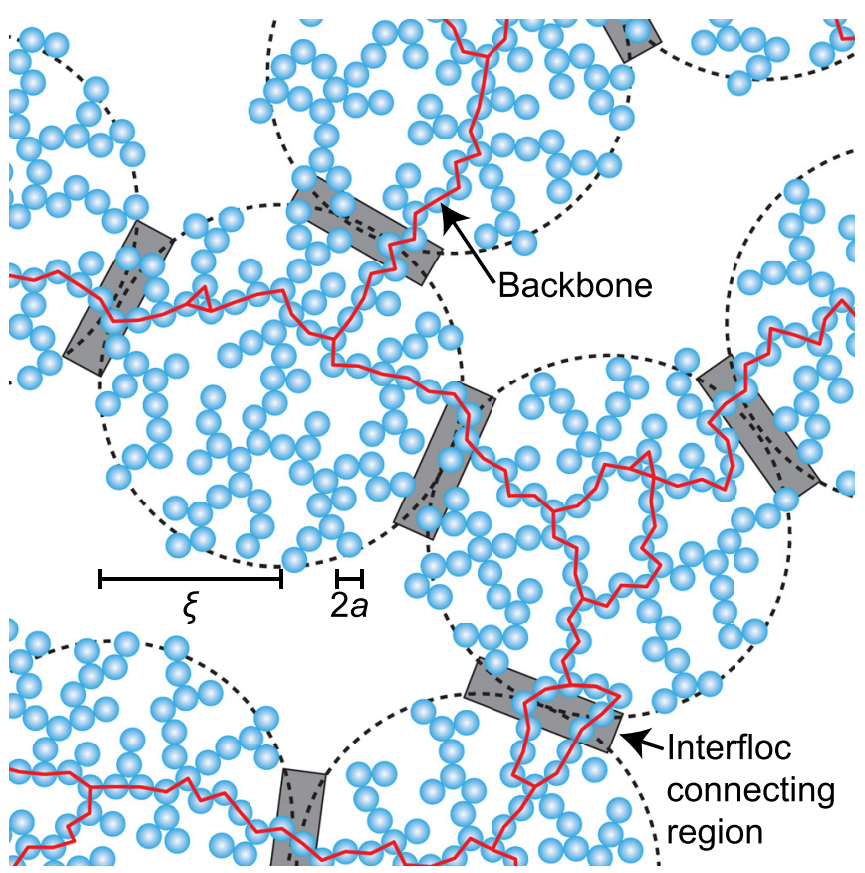

FIG. 1. Schematic drawing of a percolated network consisting of interconnected clusters (flocs) of particles having radius $a$. The large dashed-line circles denote flocs with correlation length $\xi$ and interfloc connecting regions are highlighted by the gray shaded areas. A backbone transmitting forces in the particle network is indicated by the thick red lines. While most interfloc links are part of the backbone, only a minor amount of the total particles inside of a floc contributes to the backbone.

$$
\frac{\xi}{a}=\phi_{\text {solid }}^{1 /(D-3)}
$$

which also includes changes to the correlation length with the cluster structure via the dependence on $D[42,43]$. While the cluster fractal dimension $D$ is often treated as identical to the overall percolated gel fractal dimension, such fractality only holds for length scales smaller than $\xi$ and the gel appears nonfractal and homogeneous at larger length scales [44]. Using the aforementioned approaches on larger length scales identifies an uncorrelated structure, resulting in $D=3[43,44]$.

Since the concept of fractality was introduced to characterize clustered structures of particles about three decades ago [45], a plethora of scaling theories have emerged that relate the fractal dimension to the rheological properties of attractive particle gels as a function of the particle volume fraction $\phi_{\text {solid }}$ (e.g., $\left.[36,44,46-53]\right)$. All of these scalings are of a power law type, such that

$$
X\left(\phi_{\text {solid }}\right) \propto \phi_{\text {solid }} f(D)
$$

where $X$ serves here as a placeholder for a rheological property, e.g., yield stress $\sigma_{\mathrm{y}}$ or elastic modulus plateau $G_{0}$, and the scaling theories mostly differ in the exponent $f(D)$, commonly denoted by $m$. Piau et al. [47] have proposed a model for the yield stress scaling of particle gels with

$$
f(D) \equiv m=\frac{4}{3-D} .
$$

The model of Piau et al. relates the yielding behavior of the particle gel to the free energy stored in a volume with scale $\xi$, which is not related to $\phi_{\text {solid }}$ via Eq. (4), but instead via a scaling argument following the method of de Gennes designed for mesh size determinations of semidilute polymer gels [54]. While this derivation seems to be somewhat arbitrary, Piau et al. have compared fractal dimensions calculated with their model to fractal dimensions experimentally determined by light scattering methods and found very good agreement. The Piau model has also been successfully applied to particle gels with a broad diversity of material compositions [47,55-57].

Shih et al. [46] proposed a very different model relating the network structure to the elastic properties of the particle gel, which are represented by two different elastic constants $K_{\mathrm{f}}$ and $K_{\mathrm{b}} . K_{\mathrm{f}}$ is the intrafloc constant characterizing the elasticity inside a cluster of size $\xi$ and $K_{\mathrm{b}}$ characterizes the interfloc or backbone connections between different flocs. The interfloc connecting regions are highlighted by the gray boxes in Fig. 1. Such an inhomogeneous gel structure is specifically expected for intermediate particle volume fraction ranges $\left(\phi_{\text {solid }} \approx 0.2-0.5\right)[58,59]$, which is often the relevant range for capillary suspensions. Following Shih et al., the relative contribution of both elastic constants determines the sign and slope of a scaling exponent $f(D) \equiv m_{\gamma}$ where the rheological property $X$ in Eq. (5) is the critical strain amplitude $\gamma_{\text {crit }}$ from oscillatory shear rheometry that denotes the end of the linear viscoelastic deformation regime at a given frequency. A second scaling proposed for $G_{0}$, the frequencyindependent plateau value of the elastic modulus $G^{\prime}$ in the linear viscoelastic regime, has a scaling exponent $f(D) \equiv m_{G}$. The model of Shih et al. has been further evaluated and extended by $\mathrm{Wu}$ and Morbidelli [48], who derived the two scaling exponents as

$$
\begin{aligned}
& m_{\gamma}=\frac{2-\beta}{3-D}, \\
& m_{G}=\frac{\beta}{3-D},
\end{aligned}
$$

with the parameter $\beta$ containing information about the relative contributions of intrafloc and backbone elasticities. Eliminating $\beta$ by combining Eqs. (7) and (8) leads to an expression of $D$ as a function of both scaling exponents

$$
D=\frac{3\left(m_{\gamma}+m_{G}\right)-2}{m_{\gamma}+m_{G}},
$$

where $D$, following the definitions of Eqs. (2) to (4), is the internal fractal dimension of the flocs.

The model of Piau et al., on the other hand, does not differentiate between the flocs and backbone as this theory originates from semidilute polymer network mesh sizes (i.e., small $\phi_{\text {solid }}$ for particle gels). Ergo, this model assumes the presence of a homogeneous backbone that resembles the structure of such polymer gels rather than densely packed flocs. Therefore, the corresponding fractal dimension calculated from Piau's model is not expected to be the same $D$ as calculated from the other rheological models, but should rather correspond to a "backbone dimensionality" on larger 
length scales. This implies, then, a fractal network of clusters where the clusters of size $\xi$ are the basic building blocks. Thus, $\xi$ is the lower instead of the upper length scale for the fractal structure of the network backbone [60].

\section{MATERIALS AND METHODS}

\section{A. Materials}

Commercial grade aluminum oxide particles $\left(\alpha-\mathrm{Al}_{2} \mathrm{O}_{3}\right)$ were obtained from Almatis $\mathrm{GmbH}$ (Ludwigshafen, Germany) which were used to prepare samples for rheological characterization. Four types of $\mathrm{Al}_{2} \mathrm{O}_{3}$ particles with different average CT1200SG, $a_{50,3}=0.70 \mu \mathrm{m}$; CL3000SG, $a_{50,3}=1.94 \mu \mathrm{m}$; and $\left.\mathrm{CT} 19 \mathrm{FG}, a_{50,3}=3.12 \mu \mathrm{m}\right)$. Figure 2 shows the respective particle size distributions and scanning electron microscopy (SEM) images of the particles. The particles have irregular, angular, convex shapes. Their density $\rho$ is $3.94 \mathrm{~g} / \mathrm{ml}$. The bulk phase in the $\mathrm{Al}_{2} \mathrm{O}_{3}$ material system was paraffin oil (Carl Roth, Karlsruhe, Germany). It has a Newtonian flow behavior and a particle radius $a_{50,3}$ were used (CT3000SG, $a_{50,3}=0.32 \mu \mathrm{m}$;

dynamic viscosity of $\eta\left(20^{\circ} \mathrm{C}\right)=0.029 \mathrm{~Pa}$ s. The density of the paraffin oil is $0.85 \mathrm{~g} / \mathrm{ml}$. As an appropriate secondary liquid phase, we used an aqueous sucrose solution (50 vol. \%) with $\rho\left(20^{\circ} \mathrm{C}\right)=1.28 \mathrm{~g} / \mathrm{ml}$ and $\eta\left(20^{\circ} \mathrm{C}\right)=0.080 \mathrm{~Pa}$ s. The D- $(+)-$ sucrose was obtained from Carl Roth (Karlsruhe, Germany). The secondary liquid is no solvent for the $\alpha-\mathrm{Al}_{2} \mathrm{O}_{3}$. The interfacial tension between bulk and secondary liquids is $\Gamma=41.2 \pm 0.7 \mathrm{mN} / \mathrm{m}$ as determined with the pendant drop method (OCA 15 EC, Dataphysics, Filderstadt, Germany) and the contact angle of secondary fluid toward the $\mathrm{Al}_{2} \mathrm{O}_{3}$ in bulk environment is $\theta=41 \pm 4^{\circ}$ as determined with the sessile drop method on a smooth $\alpha-\mathrm{Al}_{2} \mathrm{O}_{3}$ plate using the same apparatus as for pendant drop measurements. Thus, the system is in the pendular state.

For confocal microscopy, a different sample system based on silica particles was used. The silica particles were manufactured at Akzo Nobel (Bohus, Sweden; the smallest size donated by the manufacturer and the others purchased from MZ-Analysentechnik GmbH, Mainz, Germany). The silica particles were covalently dyed with fluorescent rhodamine B isothiocyanate via a modified Stöber synthesis [26,61,62],
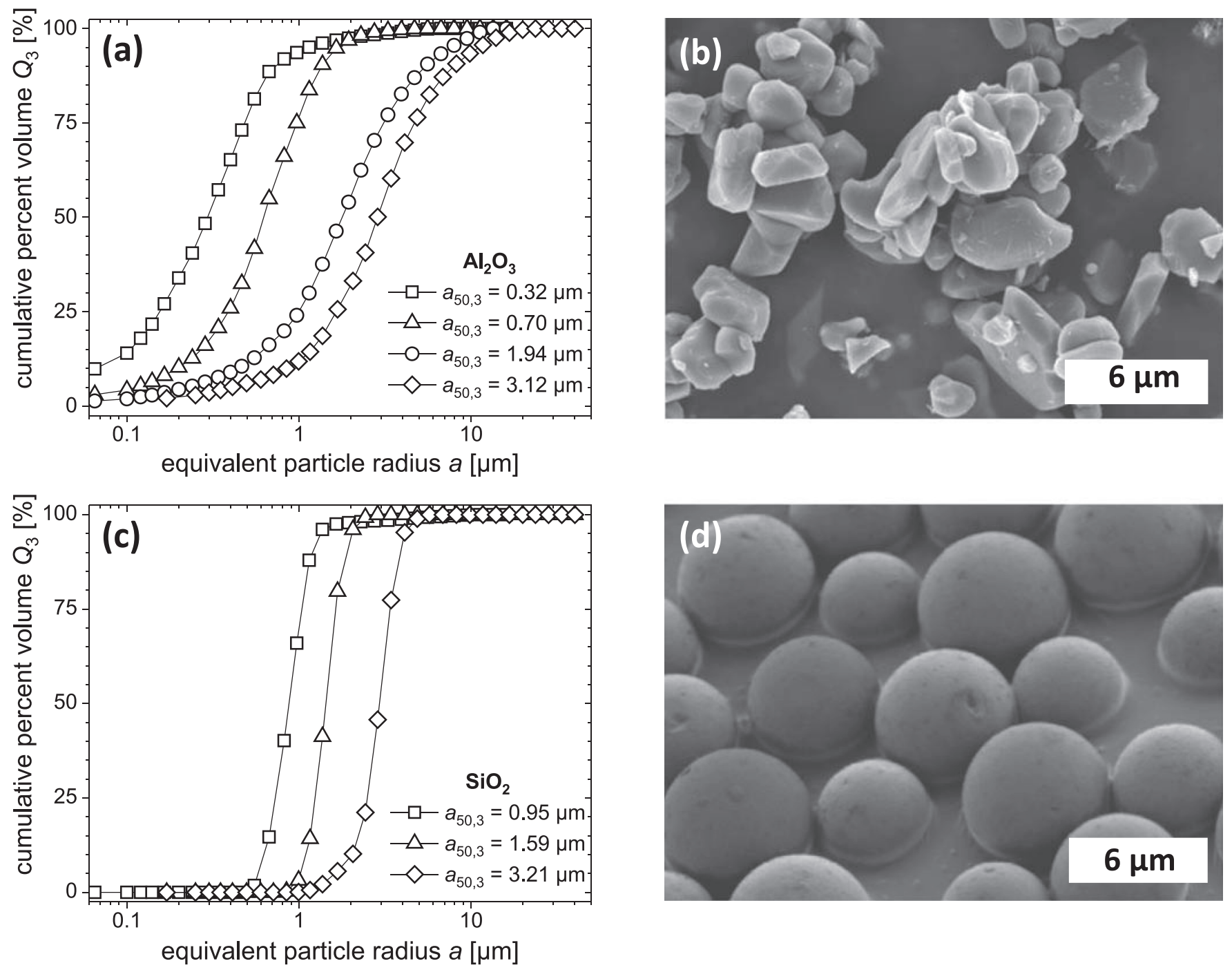

FIG. 2. Particle size distributions and $\mathrm{SEM}$ images of both particle types used in this study. (a) Particle size distribution of the different $\mathrm{Al}_{2} \mathrm{O}_{3}$ particles. (b) SEM image of $\mathrm{Al}_{2} \mathrm{O}_{3}$ with mean radius $a_{50,3}=3.12 \mu \mathrm{m}$. (c) Particle size distribution of the different silica particles. (d) SEM image of silica with $a_{50,3}=3.21 \mu \mathrm{m}$. 
and the surface was partially hydrophobized with trimethylchlorosilane [63]. Our methods to perform these chemical particle reaction steps are detailed in supplementary material, Sec. 1 [64]. Three types of silica with different mean particle radius were used (Kromasil 100-1.8-SIL, $a_{50,3}=0.95 \mu \mathrm{m}$; Kromasil 100-3.5-SIL, $a_{50,3}=1.59 \mu \mathrm{m}$; and Kromasil 100-7SIL, $a_{50,3}=3.21 \mu \mathrm{m}$; all sizes determined after chemical modifications). The dyed particles are spherical [see Fig. 2(d)] and the measured density is $1.95 \pm 0.08 \mathrm{~g} / \mathrm{ml}$.

The bulk phase in the $\mathrm{SiO}_{2}$ material system was a mixture of 86.8 wt. \% 1,2-cyclohexane dicarboxylic acid diisononyl ester (Hexamoll DINCH, BASF, Ludwigshafen, Germany) and 13.2 wt. \% n-dodecane (Alfa Aesar, Karlsruhe, Germany). Bulk mixture properties are $\rho=0.91 \mathrm{~g} / \mathrm{ml}$ and $\eta\left(20^{\circ} \mathrm{C}\right)$ $=0.021 \mathrm{~Pa}$ s. The secondary phase in the $\mathrm{SiO}_{2}$ material system was a mixture of 86.4 wt. \% glycerol (purity $>99.5 \%$, Carl Roth, Karlsruhe, Germany) and 13.6 wt. \% ultrapure water, which does not modify the silica particles. Minute amounts of the fluorescent dye PromoFluor-488 Premium carboxylic acid (PromoKine, Heidelberg, Germany) were added to the secondary fluid. Secondary fluid mixture properties are $\rho=1.23 \mathrm{~g} / \mathrm{ml}$ and $\eta\left(20^{\circ} \mathrm{C}\right)=0.120 \mathrm{~Pa}$ s. The bulk and secondary fluid mixtures had the same refractive index as the dyed silica particles $(n=1.455 \pm 0.001)$. Index matching was necessary to obtain high quality confocal images. The interfacial tension between bulk and secondary liquids, determined from pendant drop measurements, is $\Gamma=21.5 \pm 0.3 \mathrm{mN} / \mathrm{m}$. Contact angles for all three silica particle sizes were directly determined from confocal images of individual particles that are situated in an interface between bulk and secondary liquid. A detailed description of this method is given elsewhere [26]. The contact angle of secondary fluid toward the dyed silica particles in bulk environment is $\theta=65 \pm 7^{\circ}$. Thus, this system is also in the pendular state. This is a necessary precondition for assuring comparability of the silica and $\mathrm{Al}_{2} \mathrm{O}_{3}$ systems, together with the similar size ranges of both particle types. In the remainder of the paper, we will denote the mean particle radius $a_{50,3}$ simply by $a$.

\section{B. Sample preparation}

Suspensions based on $\mathrm{Al}_{2} \mathrm{O}_{3}$ particles were prepared by mixing the particles into the bulk phase with a high speed dissolver stirrer (diameter $5 \mathrm{~cm}$ ) at a rotation speed of $1200 \mathrm{rpm}$ for $10 \mathrm{~min}$. Afterward, the secondary liquid phase was added at a speed of $1000 \mathrm{rpm}$, and mixed for $2 \mathrm{~min}$. After preparing the suspensions in the high speed dissolver, a homogenization step in a self-constructed ball mill (ball diameter $20-25 \mathrm{~mm}$ ) followed. The homogenization time was $24 \mathrm{~h}$ at $18 \mathrm{rpm}$. The total sample volume was $100 \mathrm{ml}$.

The silica based capillary suspensions were prepared by emulsification of the secondary fluid in the bulk phase with an ultrasound sonifier (Ditigal Sonifier model 250, Branson Ultrasonics Corporation, Danbury, USA) with an ultrasonic horn of $12.5 \mathrm{~mm}$ diameter and maximum available output power of $200 \mathrm{~W}$. Emulsification occurred at an amplitude of $35 \%$ for $30 \mathrm{~s}$. Thereafter, the particles were stirred in with a spatula and three more ultrasonic mixing steps were applied to the sample (each $10 \mathrm{~s}$ at $10 \%$ amplitude, stirring with a spatula between steps to enhance sample homogeneity). The total sample volume was $0.85 \mathrm{ml}$. Preparation of large volumes was limited by the small total amounts of available silica particles resulting from the different chemical modification steps detailed in the supplementary material, Sec. I. These small volumes are also the reason for not using the silica system for the extensive rheological measurements, where the $\mathrm{Al}_{2} \mathrm{O}_{3}$ system was chosen instead.

\section{Rheological measurements}

Rheological behavior of suspensions based on $\mathrm{Al}_{2} \mathrm{O}_{3}$ particles was determined with steady as well as oscillatory shear measurements. For yield stress determination, the stresscontrolled rheometers MARS II (minimum torque $M_{\min }=50$ $\mathrm{nN} \mathrm{m})$ and RheoStress RS150 $\left(M_{\min }=500 \mathrm{nN}\right.$ m, both rheometers from Thermo Fisher Scientific, Karlsruhe, Germany) with a four blade vane geometry $(5 \mathrm{~mm}$ radius, $22 \mathrm{~mm}$ blade height) in an appropriate cylinder cup (Z-20-DIN, $10.8 \mathrm{~mm}$ cup radius) were used and the shear stress was increased stepwise from 1 to $10000 \mathrm{~Pa}$. The steps were logarithmically distributed with 50 steps per decade. Each step followed a dwell time of $5 \mathrm{~s}$. The capillary suspensions show transient behavior on short timescales, but the dwell time was monitored to be sufficient to reach steady state. The gels also do not demonstrate aging behavior over the experimental timescales. The yield stress was determined using the tangent method $[65,66]$. The storage modulus $G^{\prime}$ and loss modulus $G^{\prime \prime}$ were determined via oscillatory shear measurements performed on the MARS II rheometer equipped with plate-plate geometry (diameter $35 \mathrm{~mm}$, gap height $1 \mathrm{~mm}$ ). Lower steel plate and upper titanium plate surfaces were sandblasted to prevent wall slip. Stress amplitude sweep measurements were performed at angular frequencies $\omega=1 \mathrm{rad} / \mathrm{s}$ and $\omega=100 \mathrm{rad} / \mathrm{s}$. Stress controlled frequency sweep measurements were performed at a stress inside the linear viscoelastic region (sample dependent, between 10 and $150 \mathrm{~Pa}$ ) in a frequency range of $100-0.1 \mathrm{rad} / \mathrm{s}$ and show a frequency independent storage modulus of the suspensions in this range. All measurements were conducted at $20^{\circ} \mathrm{C}$. Despite the density mismatch between particles and liquids, neither particle sedimentation nor compaction of the sample during the rheological (and confocal) measurements is observed. This is due to the very strong capillary forces, which are orders of magnitude larger than the gravitational force on the particles.

\section{Confocal microscopy}

Confocal images were taken with a Leica TCS SP8 inverted confocal laser scanning microscope (Leica Microsystems, Mannheim, Germany). The microscope is equipped with two solid state lasers with wavelengths of 488 and $552 \mathrm{~nm}$. PromoFluor-488 Premium carboxylic acid dye, in the capillary suspensions' secondary phase, is excited by the $488 \mathrm{~nm}$ laser and rhodamine B isothiocyanate, in the particles, is excited by the $552 \mathrm{~nm}$ laser. The secondary phase dye emission was detected in a wavelength range of $495-520 \mathrm{~nm}$ and the particles at $650-700 \mathrm{~nm}$. A glycerol immersion objective with a numerical aperture of 1.3 and $63 \times$ magnification (HC PL 

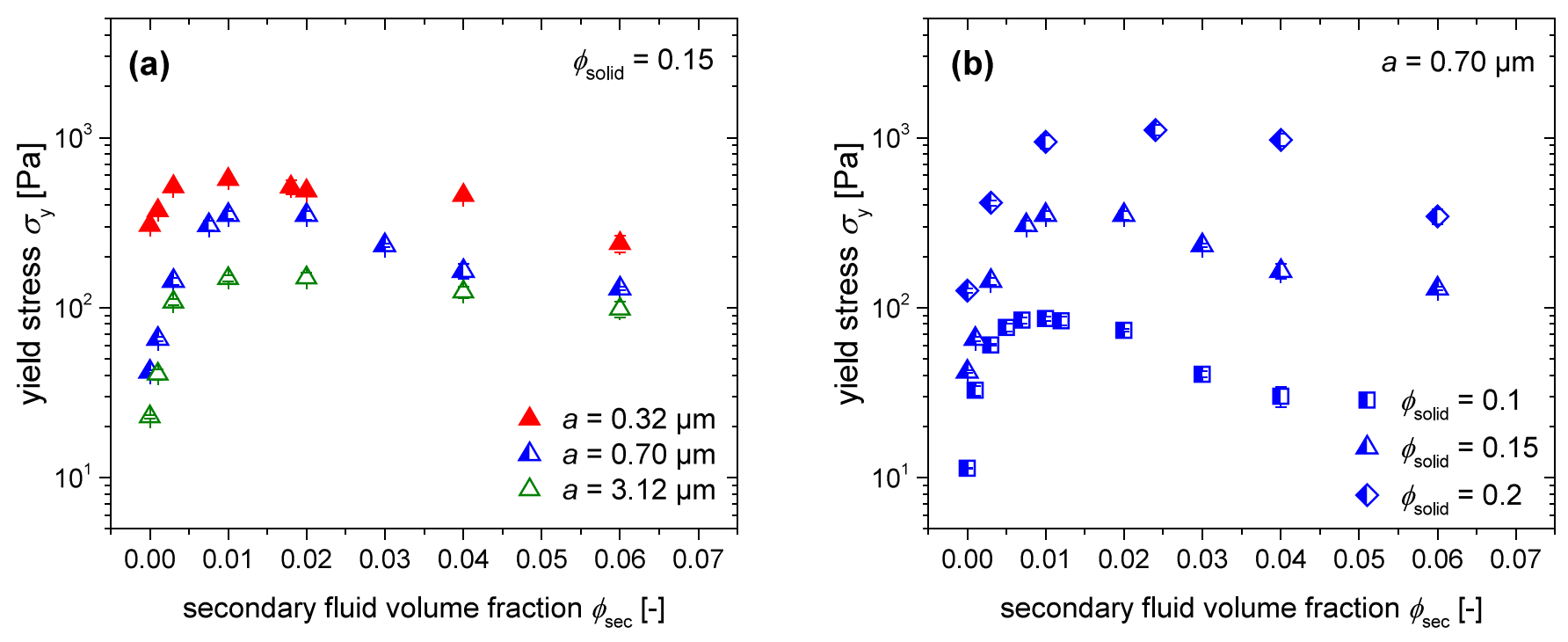

FIG. 3. Yield stress $\sigma_{\mathrm{y}}$ as function of second fluid volume fraction $\phi_{\text {sec }}$ for $\mathrm{Al}_{2} \mathrm{O}_{3}$ based capillary suspensions at (a) three different particle sizes $a$ with constant solid volume fraction $\phi_{\text {solid }}=0.15$ and (b) three different $\phi_{\text {solid }}$ with constant particle size $a=0.70 \mu \mathrm{m}$.

APO $63 \times / 1.30$ GLYC CORR CS2, Leica Microsystems, Mannheim, Germany) was used for image acquisition.

Image analysis was completed using IDL (Exelis VIS). Localization of particle positions was based on a modification of the 2D particle tracking algorithm of Crocker and Grier [67], which had been further developed for 3D detection by Weeks et al. [68]. ${ }^{1}$ Due to the size distribution and high volume fractions of particles, some more elaborate processing steps were necessary, similar to the procedure used by Wenzl et al. [69]. A detailed description of these procedures for particle localization and the subsequent determination of fractal dimensions from microscopy images are given in the supplementary material, Sec. 2.2.

\section{RESULTS AND DISCUSSION}

\section{A. Yield stress master curve for $\mathrm{Al}_{2} \mathrm{O}_{3}$ system}

The yield stress $\sigma_{\mathrm{y}}$ of various samples of the $\mathrm{Al}_{2} \mathrm{O}_{3}$ system was measured with variations of the mean particle size $a$, secondary fluid volume fraction $\phi_{\mathrm{sec}}$, and particle volume fraction $\phi_{\text {solid. }}$ Figure 3(a) shows $\sigma_{\mathrm{y}}$ as function of $\phi_{\mathrm{sec}}$ with different sizes $a$ at a constant solid volume fraction $\phi_{\text {solid }}=0.15$, while Fig. 3(b) shows $\sigma_{\mathrm{y}}$ as function of $\phi_{\mathrm{sec}}$ with varied $\phi_{\text {solid }}$ at a constant particle size $a=0.70 \mu \mathrm{m}$. In all cases, the yield stress increases strongly at first with small amounts of added second fluid due to the buildup of capillary bridges in the pendular state, then goes through a maximum, and finally $\sigma_{\mathrm{y}}$ decreases again when $\phi_{\mathrm{sec}}$ is further increased. The position of the maximum yield stress is shifted to higher second fluid volumes with increasing solid volume fraction [Fig. 3(b)], but is independent of the particle size [Fig. 3(a)]. This maximum value is typically attributed to a funicular network structure, i.e., merged capillary bridges [26,70], while the ensuing decrease occurs due to the formation of larger dense agglomerates,

${ }^{1}$ Crocker, J. C., and E. R. Weeks, "Particle tracking using IDL," http:// www.physics.emory.edu/faculty/weeks//idl/ which lead to weakening of the network structure [5,9]. The details of yield stress dependence on second fluid content have already been thoroughly investigated by Dittmann et al. on the same sample system [9], and are a general characteristics of pendular state capillary suspensions [71].

In Fig. 4, we show the same data as in Fig. 3 and include additional data taken at $\phi_{\text {solid }}=0.10$ for $a=0.32 \mu \mathrm{m}$ as well as $\phi_{\text {solid }}=0.20$ and 0.25 for $a=3.12 \mu \mathrm{m}$. These data have been rescaled so that all data collapse onto a single master curve that can be used to predict an approximate yield stress for any combination of particle size, second fluid, and solid volume fraction. While this curve is a very helpful tool for directed product design based on such capillary suspensions, it is the details of the rescaling that are of fundamental scientific interest. The rescaling on the abscissa, where $\phi_{\mathrm{sec}}$ is replaced by $\phi_{\mathrm{sec}} / \phi_{\text {solid }}$, is quite easy to understand since all

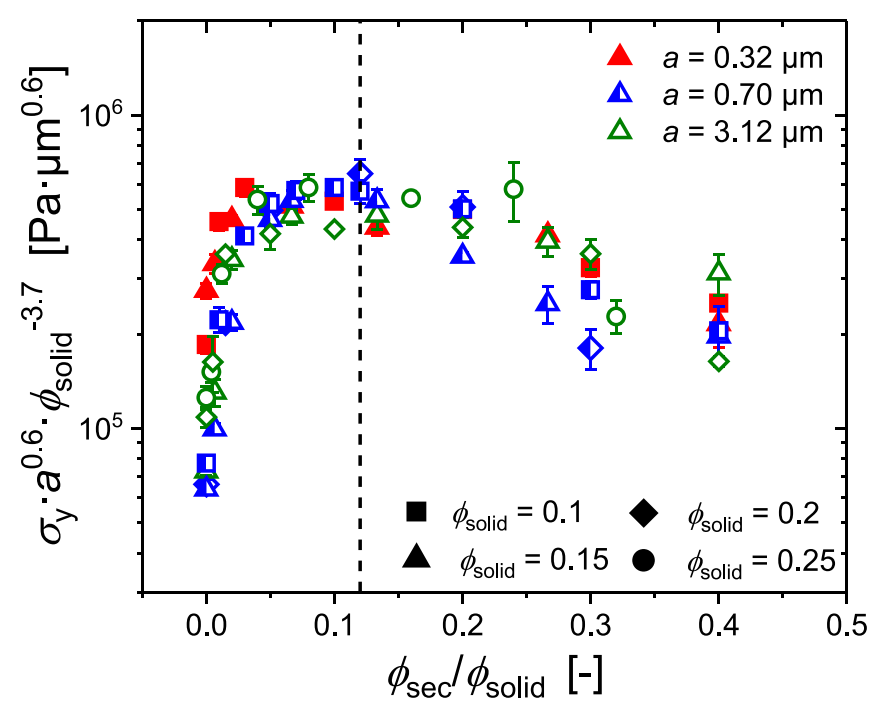

FIG. 4. Yield stress master curve for $\mathrm{Al}_{2} \mathrm{O}_{3}$ based capillary suspensions. Different symbol shapes denote each solid volume fraction and symbol fillings and color denote each particle size. The dashed line designates the position of the yield stress maximum at $\phi_{\text {sec }} / \phi_{\text {solid }}=0.12$. 
of the structural transitions, from pure suspension to the pendular state to the funicular state and finally to spherical agglomeration, depend on the relative amount of the secondary fluid droplets, characterized by the droplet size and number, to the amount of particles. This ratio is captured by the relative volume fractions with the addition of the average number of bridges per particle. Increasing, e.g., the particle volume fraction while keeping the second fluid volume fraction unchanged would either, at fixed droplet volumes, lead to a reduced number density of pendular bridges per particle contacts or, retaining the bridge number density, to a reduced volume of the bridges, and thus a different network structure or mechanical strength. Indeed, a mixed contribution of both effects is likely to appear. Accordingly, $\phi_{\text {sec }} / \phi_{\text {solid }}$ has already been used as relevant property involved in structural transitions of capillary suspensions in other studies $[5,20,66,71]$. The transition from the yield stress increase to the maximum plateau value at $\phi_{\text {sec }} / \phi_{\text {solid }} \approx 0.02-0.04$ agrees well with the ratio of single bridge to particle volumes $V_{\text {bridge }} / V_{\text {particle }}=0.021-0.025$ where the pendular-to-funicular transition was calculated for glass bead based capillary suspensions with contact angles in the range of $\theta=40^{\circ}-61^{\circ}$ [26]. Similarly, for wet granular materials with $\phi_{\text {solid }} \approx 0.57$ the upper limit of the pendular regime has been found at $\phi_{\mathrm{sec}} \approx 0.025$, denoting this transition to take place at $\phi_{\mathrm{sec}}$ $\phi_{\text {solid }} \approx 0.04$ [70].

The rescaling on the ordinate axis of Fig. 4 made by dividing the yield stress by $\phi_{\text {solid }}{ }^{3.7}$ and $a^{-0.6}$ is more complex to interpret. Using Eq. (1) as a guide, the dependence on $V_{\text {bridge }}$ is via the normalized second fluid ratio $\phi_{\text {sec }} / \phi_{\text {solid }}$ on the abscissa and $\Gamma$ and $\theta$ are constant for all samples. Therefore, $\sigma_{\mathrm{y}}$ can be rescaled by the only two remaining variables $\phi_{\text {solid }}$ and $a$. From Eq. (1), $\sigma_{\mathrm{y}}$ should then be a function of $a^{-1}$ instead of $a^{-0.6}$ and the solid volume scaling is composed of the unknown term $f\left(\phi_{\text {solid }}\right)$. Recently, $f\left(\phi_{\text {solid }}\right)=\phi_{\text {solid }}{ }^{2}$ from sparse suspensions was proposed by Koos [2], but this obviously cannot hold. A dependence of $\phi_{\text {solid }}{ }^{3.3}$ has been found by Domenech et al. for a capillary suspension system with a much lower $\theta$ [5], which corroborates the dependence of $\phi_{\text {solid }}^{3.7}$ found here. Additionally, we have performed yield stress measurements with another capillary suspension system based on $\mathrm{CaCO}_{3}$ particles, presented in the supplementary material, Sec. 3, that can also be rescaled using a solid volume fraction exponent of 3.7, which is further confirmation for the presented result.

While the theoretical size dependence of $a^{-1}$ already has been verified for capillary suspensions using glass beads [7], the deviation here might be explained by the presence of particle aggregates. It may be assumed that these aggregates rather than the individual particles form a percolated aggregate network similar to what was proposed toward the end of Sec. II. These aggregates should then form a network backbone and the yield stress is related to this network backbone rupturing. The size of the aggregates varies based on the relative droplet volume, so the exponent cannot be easily predicted and the value of -0.6 here was empirically determined. An example estimation of exponents closer to -1 using agglomerate sizes is shown in the supplementary material, Sec. 4. However, other explanations for the deviation from the $a^{-1}$ scaling are possible, e.g., a different polydispersity in particle size distributions or deviations from ideal spherical particle shape as seen in Figs. 2(a) and 2(b).

\section{B. Fractal dimension from rheological scaling laws}

For capillary suspension based product design, the maximum achievable yield stress value is often of salient interest. As can be seen in Fig. 4, this maximum appears in the $\mathrm{Al}_{2} \mathrm{O}_{3}$ system around a fixed sample composition ratio of $\phi_{\mathrm{sec}}$ $\phi_{\text {solid }}=0.12$. Figure 5 shows the yield stress at this fixed ratio as a function of the solid volume fraction for all four particle sizes. While each particle size clearly shows a power law scaling, a closer look reveals that the scaling exponent of 3.7 used for the master curve is only an inaccurate averaged value. The actual exponents $m$ increase with increasing particle sizes from $m=3.50 \pm 0.18$ at $a=0.32 \mu \mathrm{m}$ to $m=4.23 \pm 0.13$ at $a=3.12 \mu \mathrm{m}$.

In Fig. 6, the Piau model [Eq. (6)] is applied to these scaling exponents to calculate the fractal dimensions $D$ as function of the particle size. The model predicts fractal dimensions increasing from $D=1.86 \pm 0.06$ at $a=0.32 \mu \mathrm{m}$ to $D=2.05 \pm 0.03$ at $a=3.12 \mu \mathrm{m}$. These values all lie between the values of 1.85 and 2.10 reported for the DLCA and RLCA aggregation kinetic models [38]. However, one should hesitate when directly applying the DLCA and RLCA models from purely Brownian particle motion to capillary suspensions that are prepared using strong mixing (i.e., external flow fields determine particle motion) and are also influenced by gravity. In literature, hydrodynamic forces [72,73] and gravity [74-76] both have been shown to lead to rearrangements of clusters with more compact geometries with higher $D$ compared to purely diffusive aggregates, and a loss of fractality in some cases. This would imply that we should observe higher fractal dimensions $D>2.1$ that do not lie in the range between

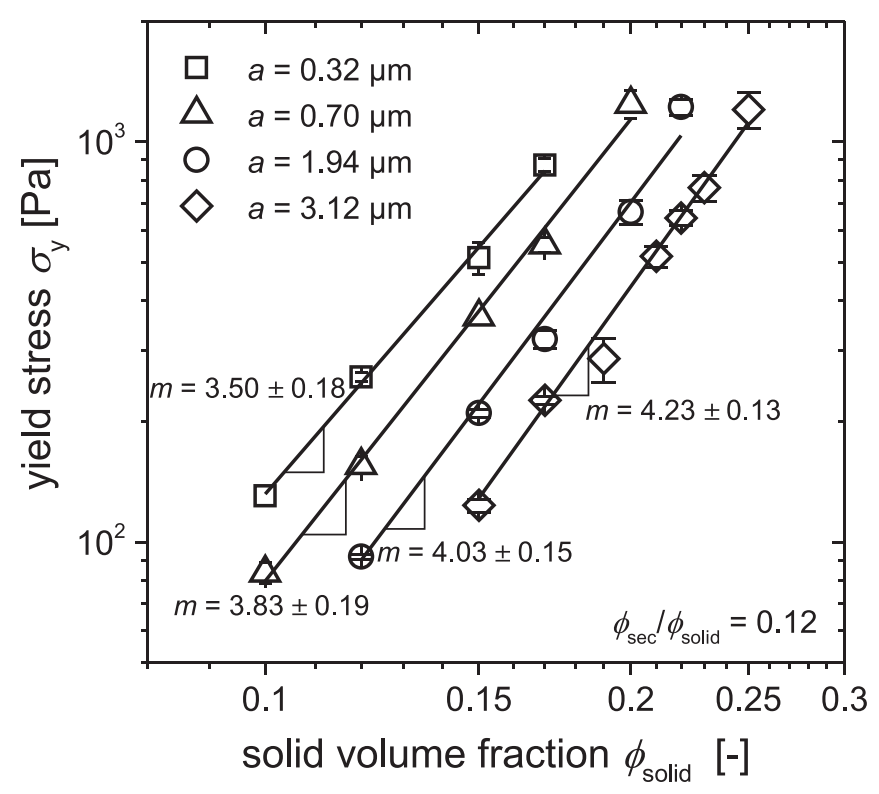

FIG. 5. Yield stress $\sigma_{\mathrm{y}}$ of $\mathrm{Al}_{2} \mathrm{O}_{3}$ based capillary suspensions as function of the solid volume fraction $\phi_{\text {solid }}$ at a fixed ratio of $\phi_{\text {sec }} / \phi_{\text {solid }}=0.12$ for the four different mean particle radii. 


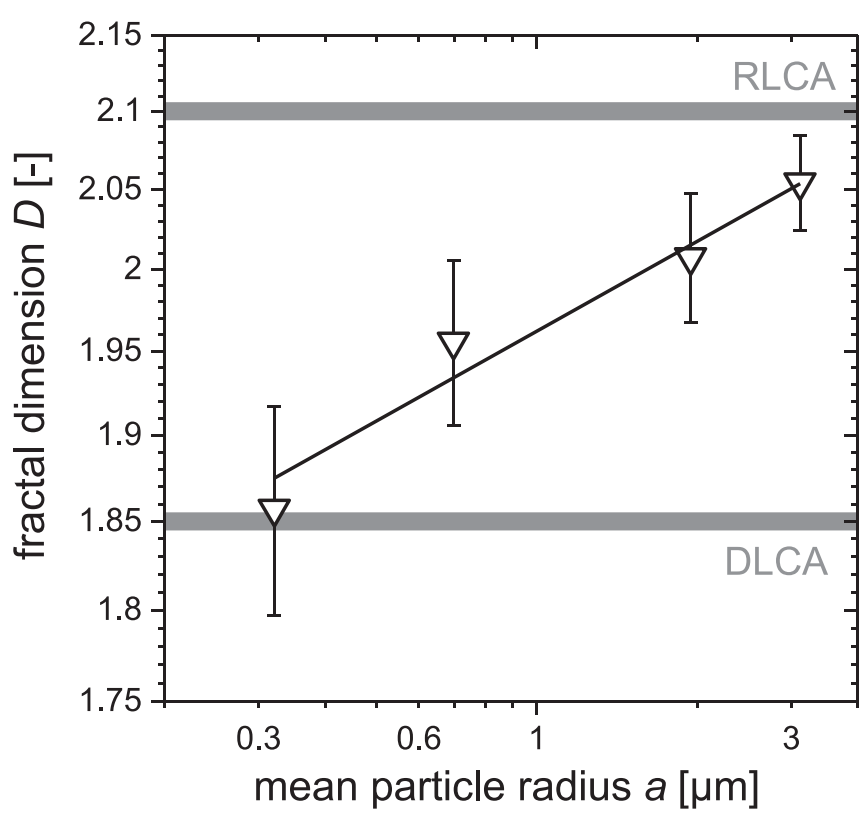

FIG. 6. Fractal dimension $D$ as function of the particle size calculated with the Piau yield stress scaling model. The gray horizontal lines denote expected fractal dimension values for DLCA and RLCA aggregation.

DLCA and RLCA values. As mentioned in the end of Sec. II, however, the Piau model is expected to predict the dimension of a network's backbone instead of the internal dimension of the basic aggregates, which may explain the calculated fractal dimensions in Fig. 6 in this range. External forces mainly influence the internal structure of the aggregates and should have less influence on the percolating backbone as this structure only obtains its final configuration once the rapid external mixing has ceased. This backbone by itself is relatively sparse compared to the interior of the aggregate, even if there is an interconnected structure. Thus, the backbone dimensionality shown in Fig. 6 should be lower than the internal aggregate dimensionality, as fractal dimensions are linked to the relative number density of particles [Eq. (3)] [43,77,78].

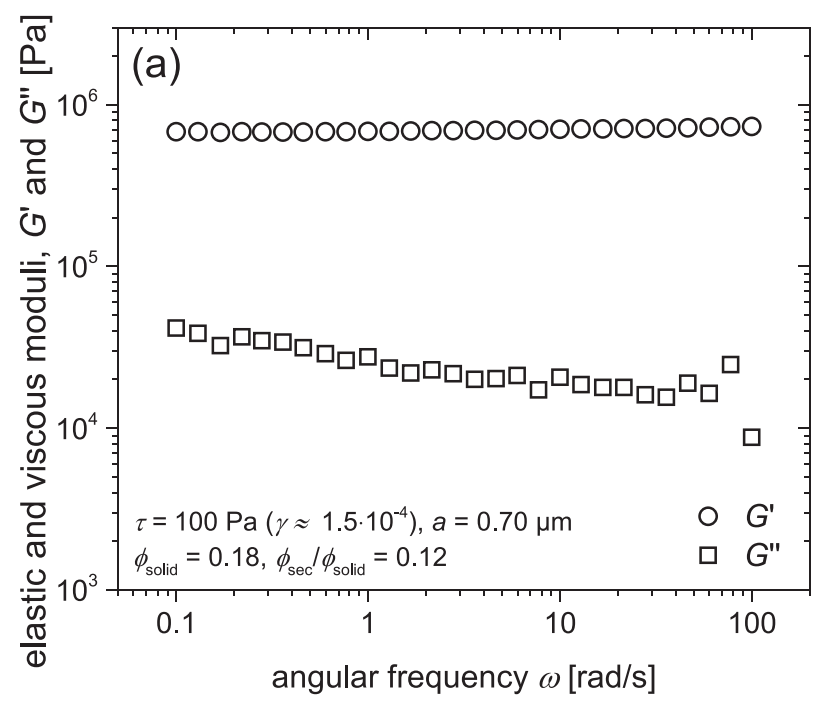

By considering the dependence of our observed dimensionalities on particle size in terms of aggregation, the sticking probability for approaching particles decreases with larger particle sizes. A hypothesis to explain this finding can be drawn when the attractive capillary force $F_{\mathrm{c}}$ is compared to other forces acting on the colliding particles. While $F_{\mathrm{c}}$ is a linear function of particle size $F_{\mathrm{c}} \sim a$ [Eq. (1)], the inertial force $F_{\mathrm{i}}$ and hydrodynamic force $F_{\mathrm{h}}$ scale as $F_{\mathrm{i}} \sim a^{3}$ and $F_{\mathrm{h}} \sim a^{2}$, respectively [28]. Thus, with increasing particle size, the ratio of the capillary to inertial and to hydrodynamic forces becomes weaker, which leads to a reduced sticking probability and thus to more compact agglomerate structures. The ratio of capillary to the other relevant forces decreases with increasing particle size independent of the mode of aggregation. Therefore, the increase in the fractal dimension for capillary suspensions with increasing particle size, seen in Fig. 6, is also expected even in the present case where the aggregation is not governed by diffusion. Further corroboration for this increase is given by yield stress data obtained with another sample system based on $\mathrm{CaCO}_{3}$ particles, which is shown in the supplementary material, Sec. 3.

It is also possible to determine the fractal dimension applying the model of $\mathrm{Wu}$ and Morbidelli to data from oscillatory shear rheometry. Figure 7 shows an example measurement of the moduli as a function of frequency as well as strain amplitude for an $\mathrm{Al}_{2} \mathrm{O}_{3}$-based capillary suspension at $\phi_{\text {sed }} / \phi_{\text {solid }}$ $=0.12$. All samples, independent of $\phi_{\text {solid }}$ and $a$, showed a frequency independent elastic modulus $G^{\prime}$ at strain amplitudes within the linear viscoelastic region for the frequency range tested [Fig. 7(a)]. Therefore, the frequency independent plateau modulus $G_{0}$ can be identified directly using an amplitude sweep as shown in Fig. 7(b). In addition to $G_{0}$, a critical strain amplitude $\gamma_{\text {crit }}$ denoting the limit of linear viscoelastic response was determined following Shih et al. where $\gamma_{\text {crit }}$ is defined using the 95\% criterion [46]. The critical amplitude showed no dependence on frequency $\omega$ at which the amplitude sweep was measured. The plateau modulus $G_{0}$ and the

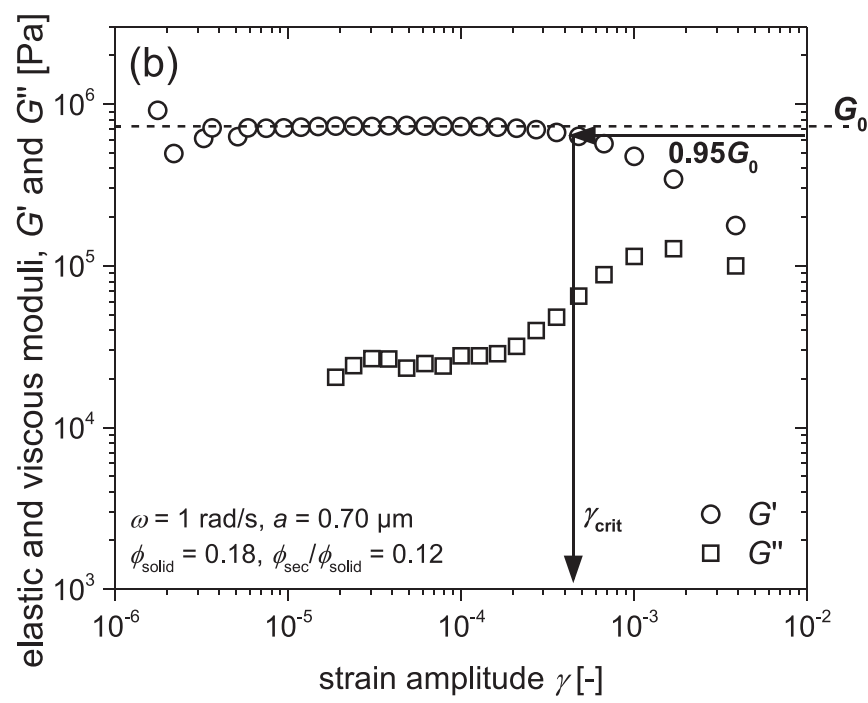

FIG. 7. Elastic and viscous shear moduli $G^{\prime}$ and $G^{\prime \prime}$ as a function of (a) angular frequency $\omega$ at a stress (and strain) amplitude inside the linear viscoelastic region and (b) strain amplitude at a frequency $\omega=1 \mathrm{rad} / \mathrm{s}$. The elastic modulus $G^{\prime}$ is shown as open circles and the viscous modulus $G^{\prime \prime}$ as open squares. The dashed line in (b) denotes the elastic plateau modulus $G_{0}$ while the arrows illustrate the determination of the critical strain amplitude $\gamma_{\text {crit }}$. Example data of an $\mathrm{Al}_{2} \mathrm{O}_{3}$-based capillary suspension with $a=0.70 \mu \mathrm{m}, \phi_{\text {solid }}=0.18$ and $\phi_{\text {sec }} / \phi_{\text {solid }}=0.12$. 
amplitude $\gamma_{\text {crit }}$ are shown in Figs. 8(a) and 8(b), respectively, as function of $\phi_{\text {solid }}$ for the four different particle sizes. $G_{0}$ can, with good agreement, be fitted by a power law with exponent $m_{\mathrm{G}}$ in the range of $3.46 \pm 0.26$ to $4.27 \pm 0.34$, but no trend with particle size is observed. The $\gamma_{\text {crit }}$ data is more scattered, but still is fit using simple power law, as often done in literature $[46,48,79]$. The exponents increase with increasing particle size from $m_{\gamma}=1.11 \pm 0.20$ at $a=0.32 \mu \mathrm{m}$ to $m_{\gamma}=4.28 \pm 1.05$ at $a=3.12 \mu \mathrm{m}$.

The fractal dimensions $D$ calculated from $m_{\mathrm{G}}$ and $m_{\gamma}$ using the model of Wu and Morbidelli [48] [Eq. (9)] are shown in Fig. 8(c) for all four particle sizes. In contrast to the fractal dimensions calculated with the Piau model from the yield stress scalings (Fig. 6), these dimensions are expected to represent the internal floc structure and not the network backbone. The values are significantly higher than the RLCA value of $D=2.1$, which due to the elevated solid volume fraction and strong external forces during sample preparation is expected. Although these dimensions differ from the ones calculated from the yield stress (Fig. 6), the trend of an increasing $D$ with particle size is reproduced. Here, the dimension ranges from $D=2.57 \pm 0.03$ at $a=0.32 \mu \mathrm{m}$ to $D=2.74 \pm 0.04$ at $a=3.12 \mu \mathrm{m}$.

Finally, it is worth noting some peculiarities in the scaling of $G_{0}$ and $\gamma_{\text {crit }}$ with particle size. First, the dependence of the plateau modulus scaling exponents, seen in Fig. 8(a), is not directly expected. While $G_{0}$ is known to often be independent of particle size for a uniform gel structure [80] this is not obvious for the heterogeneous gels investigated here. The internal floc packing geometry should depend on the particle size, especially when the agglomerating tendencies of a single particle, which was discussed at the end of Sec. IV A and is shown in Fig. S5 of the supplementary material, are kept in mind. Thus, since the fractal dimension is expected to increase, we might expect the $G_{0}$ scaling exponents to also increase with particle size. This trend is clearly shown in the scaling exponents for the critical strain [Fig. 8(b)] and for the yield stress (Fig. 5). The model from Wu and Morbidelli can help to clarify this issue. The values of $m_{\mathrm{G}}$ and $m_{\gamma}$, obtained from Figs. 8 (a) and 8(b), can be used to estimate not only $D$, but also $\beta$ using Eqs. (7) and (8). This parameter $\beta$ denotes the relative strength of inter- and intrafloc connections in the network and decreases from $\beta \approx 1.5 \pm 0.2$ at $a=0.32 \mu \mathrm{m}$ to $\beta \approx 0.9 \pm 0.2$ at $a=3.12 \mu \mathrm{m}$. A diagram of $\beta$ as function of the $\mathrm{Al}_{2} \mathrm{O}_{3}$ particle size is provided in Fig. S6 of the supplementary material. The limiting cases for $\beta$ are the pure weak-link behavior (interfloc links are much weaker than the intrafloc links), corresponding to $\beta=1$, and strong-link behavior (interfloc links are stronger than the intrafloc ones), where $\beta \approx 4.3$. These limits were first proposed in the original model of Shih et al. [46] with the intermediate values assigned only later by $\mathrm{Wu}$ and Morbidelli [48]. Therefore, the comparably low $\beta$ values in our study show that our samples are primarily dominated by weak interfloc links in all cases, but that these still become relatively weaker with increasing particle size, reaching the limit of pure weak-link behavior. The local change in packing inside of the flocs with increasing particle size (i.e., the increase in $D$ ) does not correspond to an increase in the plateau modulus since the strengthening inside of the flocs is counteracted by a weakening of the interfloc bonds. The critical strain amplitude, however, is more sensitive to the weakening of interfloc bonds, which causes the observed dependence of the corresponding scaling exponents $m_{\gamma}$ on particle size, as seen in Fig. 8(b).

\section{Structural investigations using confocal microscopy}

We can image capillary suspensions using a model system to see the differences between the compact clusters and sparse backbone. 2D confocal images of capillary suspensions based on silica particles with three different sizes $a$ and volume fraction $\phi_{\text {solid }}=0.26 \pm 0.06$ are shown in Fig. 9 . Following the results for the $\mathrm{Al}_{2} \mathrm{O}_{3}$ system, the ratio $\phi_{\text {sed }}$ $\phi_{\text {solid }}=0.12$ was also kept constant for all confocal samples. The three confocal images have been rescaled in size to allow better visual comparability so that the scale bar in each of the three images denotes a length of 40:a. These images do not show particle gels that are homogeneous at these scales, but a structure consisting of relatively compact agglomerates with large (sparsely populated) voids. Thus, the model structure shown in Fig. 1 is indeed an appropriate representation of the structure of capillary suspensions. This means that making a
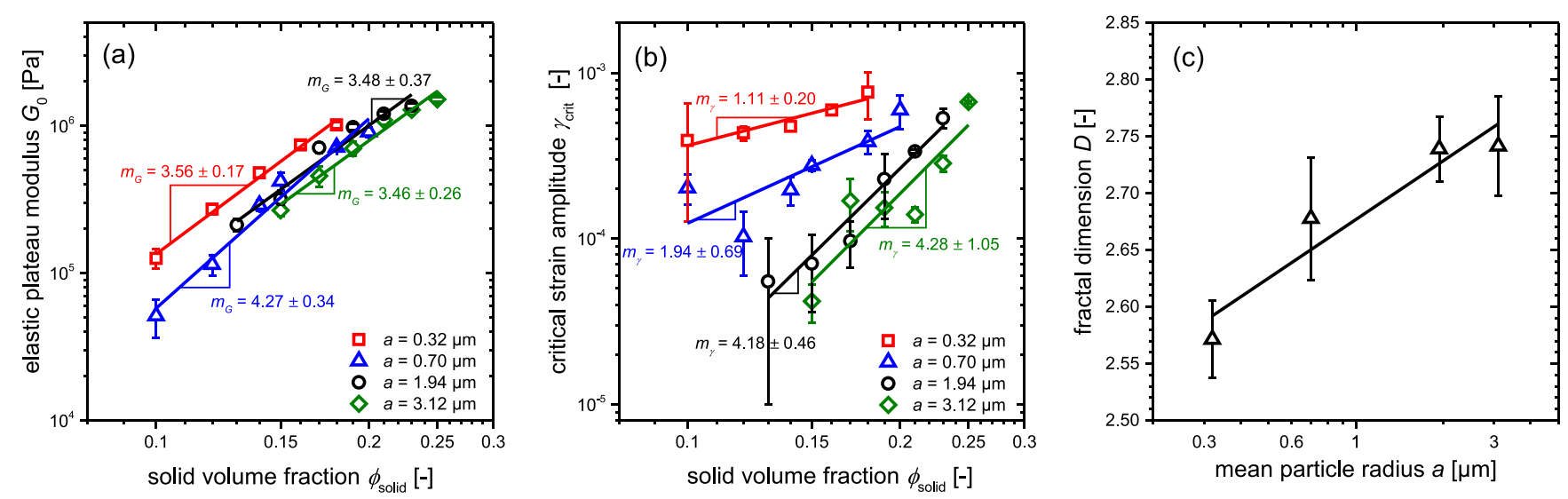

FIG. 8. (a) Elastic plateau modulus $G_{0}$ and (b) critical strain amplitude $\gamma_{\text {crit }}$ of $\mathrm{Al}_{2} \mathrm{O}_{3}$-based capillary suspensions as function of the solid volume fraction $\phi_{\text {solid }}$ at a fixed ratio of $\phi_{\mathrm{sec}} / \phi_{\mathrm{solid}}=0.12$. (c) Fractal dimensions $D$ as function of the mean particle size calculated from the data shown in (a) and (b) using the model of $\mathrm{Wu}$ and Morbidelli. 

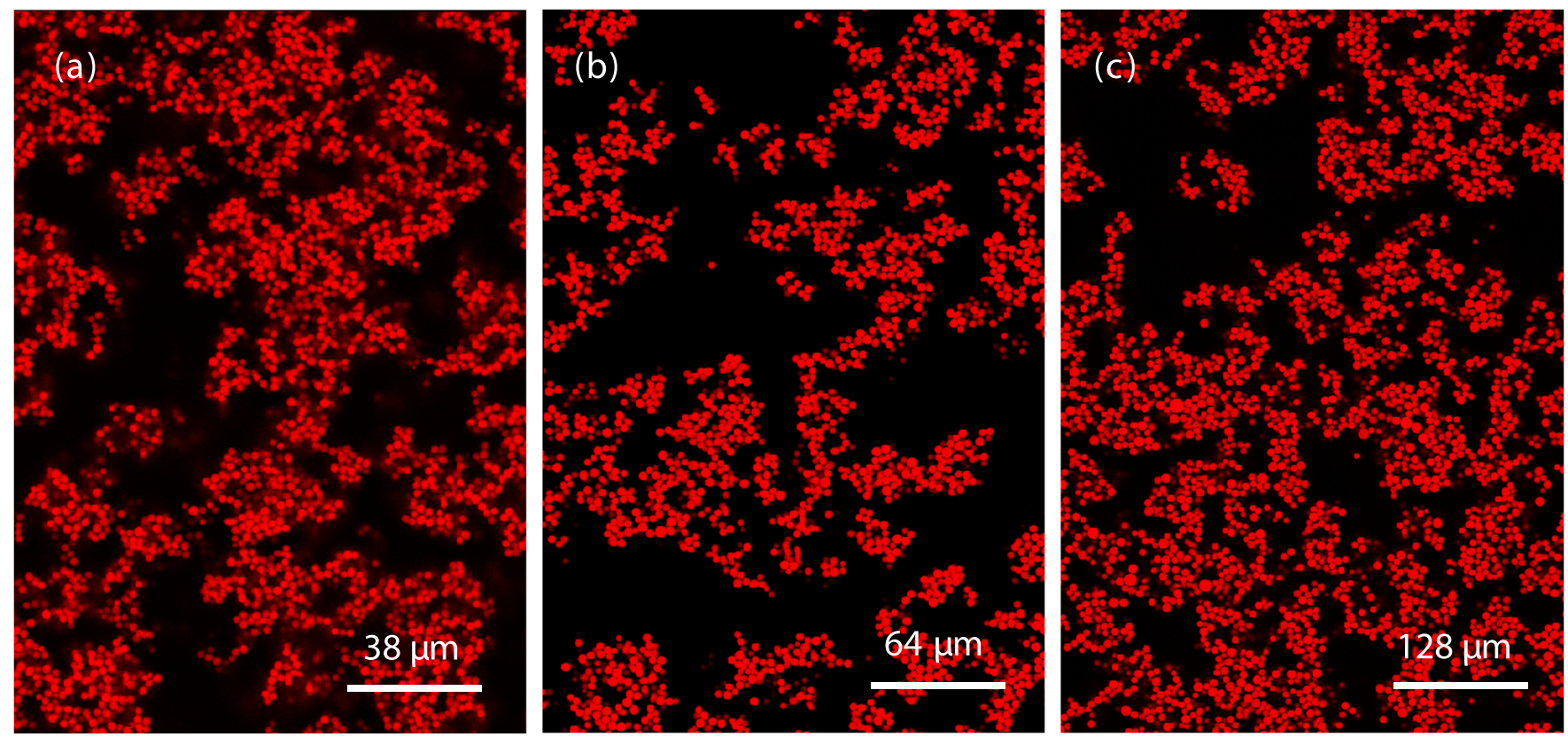

FIG. 9. Confocal 2D images of capillary suspensions based on silica particles ( $\left.\phi_{\text {solid }}=0.26 \pm 0.06\right)$ with three different mean particle radii (a) $a=0.95 \mu \mathrm{m}$, (b) $a=1.59 \mu \mathrm{m}$ and (c) $a=3.21 \mu \mathrm{m}$, all at $\phi_{\text {sed }} / \phi_{\text {solid }}=0.12$. The silica particles are shown in red. The undyed bulk liquid and secondary liquid are not shown. Image sizes and scale bars have been adjusted so that the scale bar equals $40 \cdot a$ in each image.

distinction in the strength and structure between the backbone and agglomerate obtained using the two rheological models in Sec. IV B is appropriate.

The total number of particles $N$ around a reference particle in the 3D images can be determined as function of the normalized radial distance $R / a$ to the reference particle by detecting the particle positions. An example 3D image and the method for determining the positions is described in Sec. 2 in the supplementary material. This function $N(R / a)$ can be used to calculate the fractal dimension using Eq. (3). Since the clusters have a limited size, $N(R / a)$ is not calculated for distances longer than the correlation length $\xi / a$, which is estimated from Eq. (4) using the dimension $D$ from the rheological results at first. The lowest floc dimension calculated from the oscillatory data using the $\mathrm{Wu}$ and Morbidelli model in Sec. IV B was $D \approx 2.6$. The measured $\phi_{\text {solid }}=0.26 \pm 0.06$ leads to $\xi /$ $a \approx 29 \pm 12$ (with the error in $\xi / a$ being estimated based on the error in $\phi_{\text {solid }}$ and not in $D$ ). This value roughly matches the cluster sizes visible in Fig. 9. When using the same $\phi_{\text {solid, }}$, but $D=2.0$, which is in the range predicted from the Piau model, the calculated correlation length drastically shrinks to $\xi / a \approx 3.85 \pm 0.65$. However, using $\phi_{\text {solid }}=0.26$ is incorrect in the latter calculation. While the relevant solid volume fraction for the backbone cannot be directly defined, the number of contributing particles is clearly lower than the total particle number in the sample. Thus, the relevant $\xi / a$ value for the backbone should be larger in reality.

$N(R / a)$ functions have been determined from 3D confocal images with image sizes of $(45 \cdot a)^{3}$. These images contain an average of $3300 \pm 850$ particles and the corresponding local particle volume fraction $\phi_{\text {solid }}=0.26 \pm 0.06$ was directly evaluated from the $3 \mathrm{D}$ images. Images used for fractal dimension determination have been taken at a much higher resolution than shown in Fig. 9, and the secondary fluid was also imaged.
An example image is provided in the supplementary material, Sec. 2.1. Due to the large variation in volume fraction and particle number caused by the network inhomogeneity, as seen in Fig. 9, the $N(R / a)$ function is strongly influenced by the position inside of the sample where these relatively small $3 \mathrm{D}$ images are taken. Furthermore, the arbitrarily chosen reference particles $(R=0)$ also cannot be expected to coincide with the center of gyration of a cluster. Therefore, eight such images were taken for each particle size and the $N(R / a)$ function in each image was calculated using between 350 and 550 reference particles, randomly distributed in the volume not intersecting the edges, to decrease the influence of these errors. Additionally, the maximum radial distance was set to $R /$ $a=10$, considerably lower than the estimated cluster size of $\xi /$ $a \approx 29 \pm 12$, in order to ensure many of these calculations were made inside of a cluster. The mean $N(R / a)$ functions of all these calculations are shown in Fig. 10 for the three different particle sizes.

The mean $N(R / a)$ functions show a power law increase where the exponent is evaluated for $2<R / a<10$ and the fractal dimension $D$ equals the exponent following Eq. (3). The dimensions, calculated from the image sets for each particle size, are shown in Fig. 11 as red diamonds.

This method captures mixed contributions from both the internal aggregate as well as the backbone structure as not all reference particles are close to the gyration center of the clusters, and can also include a portion of the voids, thereby reducing the effective particle density. Thus the optically determined $D$ values are larger than the calculations from the rheological scaling model of Piau, but smaller than the ones from the $\mathrm{Wu}$ and Morbidelli scaling model, representing backbone and aggregate dimensions, respectively. The inhomogeneity of the particle networks leads to scattered data in the calculations of $D$ from $N(R / a)$ functions, as local structures 


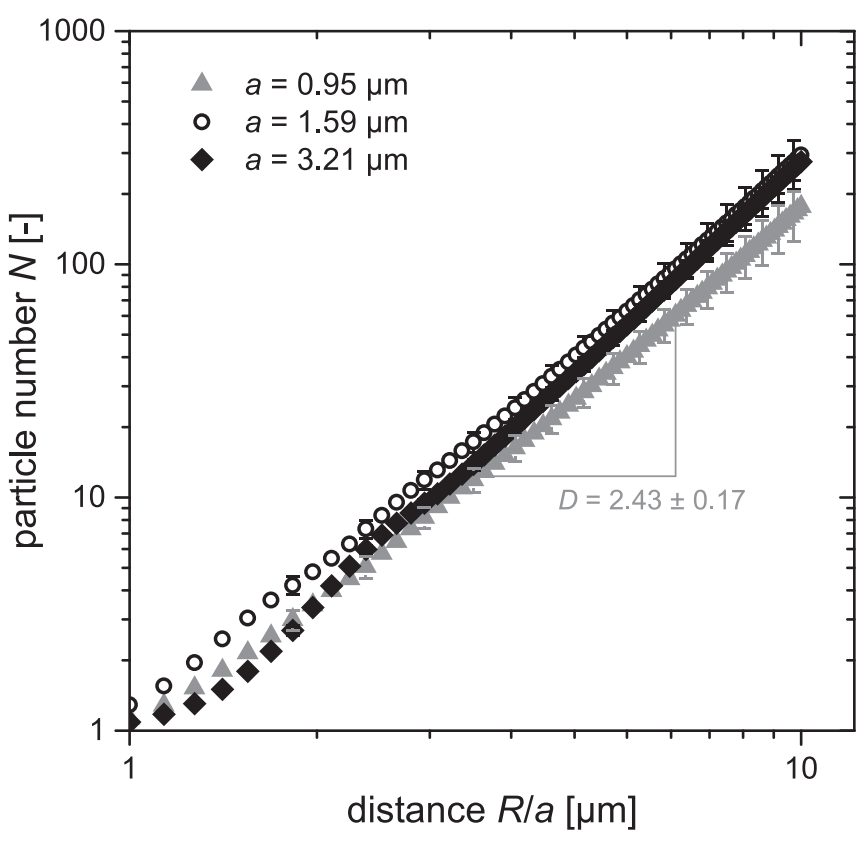

FIG. 10. Particle number function $N(R / a)$ for silica based capillary suspensions with $a=0.95 \mu \mathrm{m}$ (gray triangles), $a=1.59 \mu \mathrm{m}$ (black open circles) and $a=3.21 \mu \mathrm{m}$ (black filled diamonds), all with $\phi_{\text {solid }}=0.26 \pm 0.06$ and $\phi_{\text {sec }} /$ $\phi_{\text {solid }}=0.12 \pm 0.03$ as determined from 3D confocal image data. Error bars are only shown at every fourth data point for the sake of clarity. The exponent of the $N(R / a)$ function equals the fractal dimension $D$ as exemplified in the figure for the particles with $a=0.95 \mu \mathrm{m}$.

will vary significantly between different $3 \mathrm{D}$ images and around different reference particles. This scatter is seen in the significant size of the error bars in Figs. 10 and 11. As with the dimension determined from rheological data, the fractal dimension values from microscopy image analysis increase

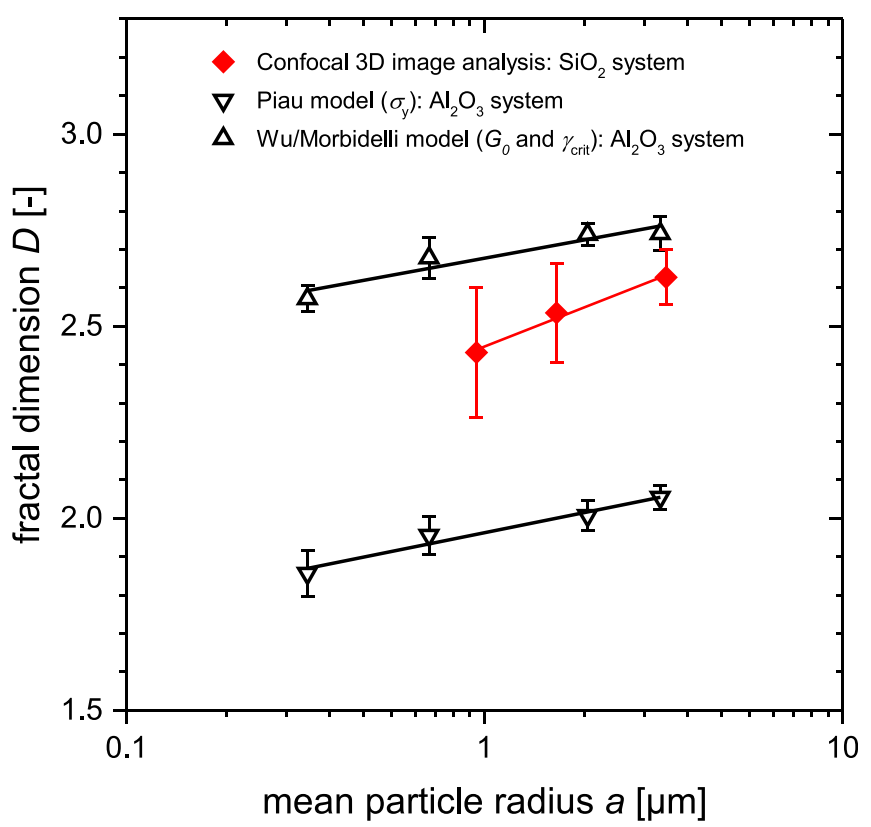

FIG. 11. Apparent fractal dimensions $D$ as function of particle size $a$ determined from confocal image analysis of silica based capillary suspensions (red diamonds). For comparison, the results from the rheological scaling models presented in Sec. IV B are reproduced in this diagram, where the backbone dimension was calculated using the model of Piau et al. and the aggregate dimension with the model of Wu and Morbidelli. with increasing particle size. This is true even though a different method and sample system were used here. There is an increase from $D=2.43 \pm 0.17$ at $a=0.95 \mu \mathrm{m}$ to $D=2.63$ \pm 0.07 at $a=3.21 \mu \mathrm{m}$. Corresponding correlation lengths can be estimated, lying between $11 \lesssim \xi / a \lesssim 38$.

Some caveats should be kept in mind when directly comparing the three curves of Fig. 11, as there are some differences between the data obtainment from rheology and from confocal microscopy. Most obvious is the usage of two sample systems, especially the difference between perfectly spherical glass beads and more irregularly shaped $\mathrm{Al}_{2} \mathrm{O}_{3}$ particles. Also, different mixing protocols had to be used to prepare the samples, which has been recently shown to influence the strength of capillary suspensions [13,32]. Closely related to the issue of mixing protocols is the general preshear history of the samples, including the loading into the measuring device for the rheometer which also differs from the microscope slide. Such different preshear protocols may also lead to different structures. Indeed, the rheological response itself might be altered by this preshear, as was shown for shear rate dependent thixotropic time scales in silica suspensions by Dullaert and Mewis [81]. Thus, comparing the absolute values of the fractal dimensions determined through confocal microscopy with the values deduced from rheology may not be valid. However, the approximate values and trends in the fractal dimensions from the different approaches are still expected to be valid. It is clear from both the rheological data and the microscopic data that these capillary suspensions exhibit a heterogeneous gel structure with fractal dimensions that increase with increasing particle size.

\section{CONCLUSIONS}

In this study, we used several approaches to determine the fractal dimension of capillary suspensions. We used rheological scaling laws that based on different structural and aggregation models and analyzed yield stress data or the elastic plateau modulus and the critical strain amplitude. These two different approaches led to different fractal dimensions capturing structural features on different length scales of the particle networks. Fractal dimensions in the range from 1.86 to 2.05 were determined using the rheological scaling model from Piau et al. [47] assuming a uniform fractal structure, while distinctly higher $D$ values in the range from 2.57 to 2.74 were obtained for the same capillary suspensions from the rheological scaling model of $\mathrm{Wu}$ and Morbidelli [48]. This marked difference is explained by an inhomogeneous microstructure of capillary suspensions, where relatively dense agglomerates are linked to form a relatively sparse backbone transmitting the forces. Taking into account the origin of the two applied rheological scaling models, the fractal dimension calculated with the Piau model is then expected to be a measure of the network backbone structure while the model from $\mathrm{Wu}$ and Morbidelli describes the fractal dimension inside the aggregates. Due to the locally higher particle volume fraction and more compact structure, the floc dimension is much higher than the backbone dimension. 
This hypothesis for interconnected dense flocs is confirmed by confocal images where particle-laden areas are separated by voids with few particles. Fractal dimensions were directly determined from particle positions obtained from 3D confocal images of index-matched capillary suspension model systems. This approach is generally more straightforward since the structural parameter $D$ is directly obtained rather than the indirect method of deducing $D$ from rheological data. While such a direct approach avoids complications and questions regarding the approximations and assumptions made to relate structure to rheology, it does not necessarily capture the inhomogeneity of the network structure. Based on structural considerations of the inhomogeneous sample structure, we indeed think it is more appropriate to not describe capillary suspension structures with only one single fractal dimension value, but to make distinctions between two different dimensionalities - one for the backbone at long length scales, and one for the internal aggregate dimensionality at short length scales. This latter value should be the parameter most closely related to the classical definition of fractal dimensions [Eq. (2)]. Due to the presence of such different length scales, the direct approach to determine a single dimension $D$ using particle number functions from microscopy also can only provide an apparent fractal dimension containing mixed contributions of both length scales. Due to the short length $R / a<10$ used to evaluate the data, the contribution of the internal cluster structure is expected to dominate, however. Accordingly, the optically determined $D$ values lie between the results obtained from the different rheological approaches, but closer to the intrafloc values from the model of $\mathrm{Wu}$ and Morbidelli than to the backbone dimensions captured by the Piau model.

Regardless of the method used, the fractal dimension of capillary suspensions increases with increasing particle size. This trend is confirmed by all of the sample systems at the different length scale provided by each method. Therefore, capillary suspensions with larger particles exhibit more compact particle arrangements. This can be explained by the weakening of the capillary force in comparison to both the inertial and hydrodynamic forces with increasing particle size. This should reduce the sticking probability of an incoming particle attaching to an aggregate during the preparation of the capillary suspension samples.

The inhomogeneous sample structure and refinement of the optically determined fractal dimension should be investigated further. In particular, our study has shown that the fractal dimension might not be the most meaningful property to get a comprehensive and complete insight into the microstructure of these capillary suspensions due to their heterogeneous nature. Other characterization approaches provide additional options that can be combined with the fractal dimension to get structural information for such heterogeneous, high volume fraction particle gels. One promising method is that of Wyss et al., who evaluated a similar heterogeneous particle gel by describing it as two-component system with one phase being represented by large voids of bulk liquid and the second component by the compact particle gel (aggregates and backbone) [59]. This second component is described by a solid volume fraction $\phi_{2}$ that is larger than $\phi_{\text {solid }}$ and is calculated as $\phi_{2}=\phi_{\text {solid }} /\left(1-\phi_{1}\right)$ with $\phi_{1}$ representing the estimated volume fraction of the void pores of bulk liquid. While it is difficult to precisely determine $\phi_{1}$, it nevertheless should be possible to estimate this value from confocal images in future studies and then determine local aggregate structures represented by $\phi_{2}$. A second promising method to characterize heterogeneous suspensions, first introduced by Varadan and Solomon, is based on the determination of Voronoi volumes around the individual particles. The broadness and the skewness of the distribution of Voronoi volumes can be used to identify the degree of structural heterogeneity [82]. One can also use other parameters to describe the network structure, such as the coordination number between particles evaluated from the confocal images [83] and then make distinctions between the particle contacts with and without liquid bridges. Similarly, obtaining a distribution of clustering coefficients might be a worthwhile approach to the local microstructure. Concerning rheological methods, detections of space-resolved stress distributions could help in fathoming details of the yielding mechanism, as yielding of heterogeneous gels can occur by only very local breaking of a very small fraction of the network connections [84]. This might also help to better explain the relative importance and strength of inter- and intrafloc bonds, which has emerged in the discussion of Fig. 8. Finally, another promising approach could be to perform simulations of the network structure and compare these simulations to optically determined data. This approach would allow structural information to be determined as sample conditions are independently varied. Thus, while we have gained interesting information about the structure of capillary suspensions by using the fractal approaches in this study, there remain many additional options for promising future research studies.

\section{ACKNOWLEDGMENTS}

The authors thank Almatis GmbH (Ludwigshafen, Germany) and Akzo Nobel (Bohus, Sweden) for donating the aluminum oxide and small-sized Kromasil silica, respectively. Irene Natalia is thanked for her very helpful collaboration and discussions on the dyeing reaction of silica particles. Additionally, the authors would like to acknowledge financial support from the German Research Foundation (Deutsche Forschungsgemeinschaft Grant No. Wi 3138/19-1) and the European Research Council under the European Union's Seventh Framework Program (FP/2007-2013)/ERC Grant Agreement No. 335380. The authors declare no competing financial, personal or other interests.

\section{References}

[1] Koos, E., and N. Willenbacher, "Capillary forces in suspension rheology," Science 331, 897-900 (2011).

[2] Koos, E., "Capillary suspensions: Particle networks formed through the capillary force," Curr. Opin. Colloid Interface Sci. 19, 575-684 (2014).

[3] van Kao, S., L. E. Nielsen, and C. T. Hill, "Rheology of concentrated suspensions of spheres. II. Suspensions agglomerated by an immiscible second liquid," J. Colloid Interface Sci. 53, 367-373 (1975). 
[4] McCulfor, J., P. Himes, and M. R. Anklam, "The effects of capillary forces on the flow properties of glass particle suspensions in mineral oil,” AIChE J. 57, 2334-2340 (2011).

[5] Domenech, T., and S. S. Velankar, "On the rheology of pendular gels and morphological developments in paste-like ternary systems based on capillary attraction," Soft Matter 11, 1500-1516 (2015).

[6] Velankar, S., "A non-equilibrium state diagram for liquid/fluid/particle mixtures," Soft Matter 11, 8393-8403 (2015).

[7] Koos, E., J. Johannsmeier, L. Schwebler, and N. Willenbacher, "Tuning suspension rheology using capillary forces," Soft Matter $\mathbf{8}$, 6620-6628 (2012).

[8] Dittmann, J., E. Koos, and N. Willenbacher, "Ceramic capillary suspensions: Novel processing route for macroporous ceramic materials," J. Am. Ceram. Soc. 96, 391-397 (2013).

[9] Dittmann, J., and N. Willenbacher, "Micro structural investigations and mechanical properties of macro porous ceramic materials from capillary suspensions," J. Am. Ceram. Soc. 97, 3787-3792 (2014).

[10] Maurath, J., J. Dittmann, N. Schultz, and N. Willenbacher, "Fabrication of highly porous glass filters using capillary suspension processing," Sep. Purif. Technol. 149, 470-478 (2015).

[11] Dittmann, J., J. Maurath, B. Bitsch, and N. Willenbacher, "Highly porous materials with unique mechanical properties from smart capillary suspensions," Adv. Mater. 28, 1689-1696 (2016).

[12] Hauf, K., K. Riazi, N. Willenbacher, and E. Koos, "Radical polymerization of capillary bridges between micron-sized particles in liquid bulk phase as a low temperature route to produce porous solid materials," Colloid Polym. Sci. 295, 1773-1785 (2017).

[13] Domenech, T., and S. Velankar, "Capillary-driven percolating networks in ternary blends of immiscible polymers and silica particles," Rheol. Acta 53, 593-605 (2014).

[14] Heidlebaugh, S., T. Domenech, S. Iasella, and S. S. Velankar, "Aggregation and separation in ternary particle/oil/water systems with fully-wettable particles," Langmuir 30, 63-74 (2014).

[15] Dapeng, W., X. Wang, Y. Yuan, W. Li, H. Tian, and S. Zhao, "Increasing the apparent shear viscosity of polymer composites by uptake of a small amount of water," RSC Adv. 4, 24686-24691 (2014).

[16] Bitsch, B., J. Dittmann, M. Schmitt, P. Scharfer, W. Schabel, and N. Willenbacher, "A novel slurry concept for the fabrication of lithiumion battery electrodes with beneficial properties," J. Power Sources 265, 81-90 (2014).

[17] Bitsch, B., T. Gallasch, M. Schroeder, M. Börner, M. Winter, and N. Willenbacher, "Capillary suspensions as beneficial formulation concept for high energy density Li-ion battery electrodes," J. Power Sources 328, 114-123 (2016).

[18] Schneider, M., E. Koos, and N. Willenbacher, "Highly conductive, printable pastes from capillary suspensions," Sci. Rep. 6, 31367 (2016).

[19] Schneider, M., J. Maurath, S. Fischer, M. Weiß, N. Willenbacher, and E. Koos, "Suppressing crack formation in particulate systems by utilizing capillary forces," ACS Appl. Mater. Interfaces 9, 11095-11105 (2017).

[20] Sun, H., X. Zhang, and M. M. Yuen, "Enhanced conductivity induced by attractive capillary force in ternary conductive adhesive," Compos. Sci. Technol. 137, 109-117 (2016).

[21] Jampolski, L., A. Sänger, T. Jakobs, G. Guthausen, T. Kolb, and N. Willenbacher, "Improving the processability of coke water slurries for entrained flow gasification," Fuel 185, 102-111 (2016).

[22] Hoffmann, S., E. Koos, and N. Willenbacher, "Using capillary bridges to tune stability and flow behavior of food suspensions," Food Hydrocolloids 40, 44-52 (2014).
[23] Wollgarten, S., C. Yuce, E. Koos, and N. Willenbacher, "Tailoring flow behavior and texture of water based cocoa suspensions," Food Hydrocolloids 52, 167-174 (2016).

[24] Maurath, J., and N. Willenbacher, "3D printing of open-porous cellular ceramics with high specific strength," J. Eur. Ceram. Soc. 37, 4833-4842 (2017).

[25] Roh, S., D. P. Parekh, B. Bharti, S. D. Stoyanov, and O. D. Velev, "3D printing by multiphase silicone/water capillary inks," Adv. Mater. 29, 1701554 (2017).

[26] Bossler, F., and E. Koos, "Structure of particle networks in capillary suspensions with wetting and non-wetting fluids," Langmuir 32, 1489-1501 (2016).

[27] Mitarai, N., and F. Nori, "Wet granular materials," Adv. Phys. 55, $1-45$ (2006).

[28] Herminghaus, S., Wet Granular Matter: A Truly Complex Fluid (World Scientific, Singapore, 2013).

[29] Willett, C. D., M. J. Adams, S. A. Johnson, and J. P. Seville, "Capillary bridges between two spherical bodies," Langmuir 16, 9396-9405 (2000).

[30] Strauch, S., and S. Herminghaus, "Wet granular matter: A truly complex fluid," Soft Matter 8, 8271-8280 (2012).

[31] Megias-Alguacil, D., and L. J. Gauckler, "Capillary forces between two solid spheres linked by a concave liquid bridge: Regions of existence and forces mapping," AIChE J. 55, 1103-1109 (2009).

[32] Bossler, F., L. Weyrauch, R. Schmidt, and E. Koos, "Influence of mixing conditions on the rheological properties and structure of capillary suspensions," Colloids Surf., A 518, 85-97 (2017).

[33] Butt, H.-J., "Controlling the flow of suspensions," Science 331, 868-869 (2011).

[34] Jullien, R., "Aggregation phenomena and fractal aggregates," Contemp. Phys. 28, 477-493 (1987).

[35] Witten, Jr., T., and L. M. Sander, "Diffusion-limited aggregation, a kinetic critical phenomenon," Phys. Rev. Lett. 47, 1400-1403 (1981).

[36] De Rooij, R., A. Potanin, D. Van den Ende, and J. Mellema, "Steady shear viscosity of weakly aggregating polystyrene latex dispersions," J. Chem. Phys. 99, 9213-9223 (1993).

[37] Kolb, M., and R. Jullien, "Chemically limited versus diffusion limited aggregation,” J. Phys. Lett. 45, 977-981 (1984).

[38] Lin, M., H. Lindsay, D. Weitz, R. Ball, R. Klein, and P. Meakin, "Universality in colloid aggregation," Nature 339, 360-362 (1989).

[39] Sonntag, R. C., and W. B. Russel, "Structure and breakup of flocs subjected to fluid stresses: I. Shear experiments," J. Colloid Interface Sci. 113, 399-413 (1986).

[40] Krall, A., and D. Weitz, "Internal dynamics and elasticity of fractal colloidal gels,” Phys. Rev. Lett. 80, 778-781 (1998).

[41] Dinsmore, A. D., E. R. Weeks, V. Prasad, A. C. Levitt, and D. A. Weitz, "Three-dimensional confocal microscopy of colloids," Appl. Opt. 40, 4152-4159 (2001).

[42] Mobuchon, C., P. J. Carreau, and M.-C. Heuzey, "Structural analysis of non-aqueous layered silicate suspensions subjected to shear flow," J. Rheol. 53, 1025-1048 (2009).

[43] Mellema, M., J. Van Opheusden, and T. Van Vliet, "Relating colloidal particle interactions to gel structure using Brownian dynamics simulations and the Fuchs stability ratio," J. Chem. Phys. 111, 6129-6135 (1999).

[44] Bremer, L. G., T. van Vliet, and P. Walstra, "Theoretical and experimental study of the fractal nature of the structure of casein gels," J. Chem. Soc., Faraday Trans. 1 85, 3359-3372 (1989).

[45] Jullien, R., and R. Botet, Aggregation and Fractal Aggregates (World Scientific, Singapore, 1987). 
[46] Shih, W.-H., W. Y. Shih, S.-I. Kim, J. Liu, and I. A. Aksay, "Scaling behavior of the elastic properties of colloidal gels," Phys. Rev. A 42, 4772-4779 (1990).

[47] Piau, J., M. Dorget, and J. Palierne, "Shear elasticity and yield stress of silica-silicone physical gels: Fractal approach," J. Rheol. 43, 305-314 (1999).

[48] Wu, H., and M. Morbidelli, "A model relating structure of colloidal gels to their elastic properties," Langmuir 17, 1030-1036 (2001).

[49] Xi, Y., J. Chen, Y. Xu, F. Chu, and C. Liu, "Yield stress of fractal aggregates," Fractals 23, 1550028 (2015).

[50] Uriev, N., and I. Y. Ladyzhinsky, "Fractal models in rheology of colloidal gels," Colloids Surf., A 108, 1-11 (1996).

[51] Studart, A. R., E. Amstad, and L. J. Gauckler, "Yielding of weakly attractive nanoparticle networks," Soft Matter 7, 6408-6412 (2011).

[52] Shih, W. Y., W. H. Shih, and I. A. Aksay, "Elastic and yield behavior of strongly flocculated colloids," J. Am. Ceram. Soc. 82, 616-624 (1999).

[53] Wessel, R., and R. Ball, "Fractal aggregates and gels in shear flow," Phys. Rev. A 46, R3008-R3011 (1992).

[54] De Gennes, P.-G., Scaling Concepts in Polymer Physics (Cornell University, Ithaca, 1979).

[55] Saint-Michel, F., F. Pignon, and A. Magnin, "Fractal behavior and scaling law of hydrophobic silica in polyol," J. Colloid Interface Sci. 267, 314-319 (2003).

[56] Loiseau, A., and J.-F. Tassin, "Model nanocomposites based on laponite and poly (ethylene oxide): Preparation and rheology," Macromolecules 39, 9185-9191 (2006).

[57] Gareche, M., A. Allal, N. Zeraibi, F. Roby, N. Azril, and L. Saoudi, "Relationship between the fractal structure with the shear complex modulus of montmorillonite suspensions," Appl. Clay Sci. 123, 11-17 (2016).

[58] Zaccone, A., H. Wu, and E. Del Gado, "Elasticity of arrested shortranged attractive colloids: Homogeneous and heterogeneous glasses," Phys. Rev. Lett. 103, 208301 (2009).

[59] Wyss, H. M., E. Tervoort, L. P. Meier, M. Müller, and L. J. Gauckler, "Relation between microstructure and mechanical behavior of concentrated silica gels," J. Colloid Interface Sci. 273, 455-462 (2004).

[60] Pignon, F., A. Magnin, J.-M. Piau, B. Cabane, P. Lindner, and O. Diat, "Yield stress thixotropic clay suspension: Investigations of structure by light, neutron, and x-ray scattering," Phys. Rev. E 56, 3281-3289 (1997).

[61] Stöber, W., A. Fink, and E. Bohn, "Controlled growth of monodisperse silica spheres in the micron size range," J. Colloid Interface Sci. 26, 62-69 (1968).

[62] Nozawa, K., H. Gailhanou, L. Raison, P. Panizza, H. Ushiki, E. Sellier, J. Delville, and M. Delville, "Smart control of monodisperse Stöber silica particles: effect of reactant addition rate on growth process," Langmuir 21, 1516-1523 (2005).

[63] Fuji, M., H. Fujimori, T. Takei, T. Watanabe, and M. Chikazawa, "Wettability of glass-bead surface modified by trimethylchlorosilane," J. Phys. Chem. B 102, 10498-10504 (1998).

[64] See supplementary material at https://doi.org/10.1122/1.4997889 for additional information on silica particle preparations, on confocal $3 \mathrm{D}$ image evaluation, additional rheological data using $\mathrm{CaCO}_{3}$-based systems, the influence of particle agglomeration, and the relative strength of inter- and intrafloc links.

[65] Brummer, R., Rheology Essentials of Cosmetic and Food Emulsions (Springer Science \& Business Media, Berlin, 2006).

[66] Maurath, J., B. Bitsch, Y. Schwegler, and N. Willenbacher, "Influence of particle shape on the rheological behavior of three-phase non-brownian suspensions," Colloids Surf., A 497, 316-326 (2016).

[67] Crocker, J. C., and D. G. Grier, "Methods of digital video microscopy for colloidal studies," J. Colloid Interface Sci. 179, 298-310 (1996).

[68] Weeks, E. R., J. C. Crocker, A. C. Levitt, A. Schofield, and D. A. Weitz, "Three-dimensional direct imaging of structural relaxation near the colloidal glass transition," Science 287, 627-631 (2000).

[69] Wenzl, J., R. Seto, M. Roth, H.-J. Butt, and G. K. Auernhammer, "Measurement of rotation of individual spherical particles in cohesive granulates," Granular Matter 15, 391-400 (2012).

[70] Scheel, M., R. Seemann, M. Brinkmann, M. Di Michiel, A. Sheppard, B. Breidenbach, and S. Herminghaus, "Morphological clues to wet granular pile stability,” Nat. Mater. 7, 189-193 (2008).

[71] Yang, J., and S. S. Velankar, "Preparation and yielding behavior of pendular network suspensions," J. Rheol. 61, 217-228 (2017).

[72] Torres, F. E., W. B. Russel, and W. R. Schowalter, "Floc structure and growth kinetics for rapid shear coagulation of polystyrene colloids," J. Colloid Interface Sci. 142, 554-574 (1991).

[73] Lin, M., R. Klein, H. Lindsay, D. Weitz, R. Ball, and P. Meakin, "The structure of fractal colloidal aggregates of finite extent," J. Colloid Interface Sci. 137, 263-280 (1990).

[74] Allain, C., M. Cloitre, and F. Parisse, "Settling by cluster deposition in aggregating colloidal suspensions," J. Colloid Interface Sci. 178, 411-416 (1996).

[75] Allain, C., M. Cloitre, and M. Wafra, "Aggregation and sedimentation in colloidal suspensions,” Phys. Rev. Lett. 74, 1478-1481 (1995).

[76] Peltomäki, M., E. Hellén, and M. Alava, "No self-similar aggregates with sedimentation,” J. Stat. Mech.: Theory Exp. 2004, P09002 (2004).

[77] Lach-Hab, M., A. E. González, and E. Blaisten-Barojas, "Concentration dependence of structural and dynamical quantities in colloidal aggregation: computer simulations," Phys. Rev. E 54, 5456-5462 (1996).

[78] Au, P.-I., J. Liu, and Y.-K. Leong, "Yield stress and microstructure of washed oxide suspensions at the isoelectric point: experimental and model fractal structure," Rheol. Acta 55, 847-856 (2016).

[79] Ikeda, S., E. A. Foegeding, and T. Hagiwara, "Rheological study on the fractal nature of the protein gel structure," Langmuir 15, 8584-8589 (1999).

[80] Larson, R. G., The Structure and Rheology of Complex Fluids (Oxford University, Oxford, 1999).

[81] Dullaert, K., and J. Mewis, "Thixotropy: Build-up and breakdown curves during flow, 'J. Rheol. 49, 1213-1230 (2005).

[82] Varadan, P., and M. J. Solomon, "Direct visualization of long-range heterogeneous structure in dense colloidal gels," Langmuir 19, 509-512 (2003).

[83] Dinsmore, A., and D. Weitz, "Direct imaging of three-dimensional structure and topology of colloidal gels," J. Phys.: Condens. Matter 14, 7581-7597 (2002).

[84] Colombo, J., and E. Del Gado, "Stress localization, stiffening, and yielding in a model colloidal gel," J. Rheol. 58, 1089-1116 (2014). 